\title{
Microrheology of colloidal dispersions: Shape matters
}

\author{
Aditya S. Khair ${ }^{\text {a) }}$ and John F. Brady \\ Division of Chemistry and Chemical Engineering, California Institute \\ of Technology, Pasadena, California 91125
}

(Received 17 January 2007; final revision received 15 November 2007)

\begin{abstract}
Synopsis
We consider a "probe" particle translating at constant velocity through an otherwise quiescent dispersion of colloidal "bath" particles, as a model for particle-tracking microrheology experiments in the active (nonlinear) regime. The probe is a body of revolution with major and minor semiaxes $a$ and $b$, respectively, and the bath particles are spheres of radii $b$. The probe's shape is such that when its major or minor axis is the axis of revolution the excluded-volume, or contact, surface between the probe and a bath particle is a prolate or oblate spheroid, respectively. The moving probe drives the microstructure of the dispersion out of equilibrium; counteracting this is the Brownian diffusion of the bath particles. For a prolate or oblate probe translating along its symmetry axis, we calculate the nonequilibrium microstructure to first order in the volume fraction of bath particles and over the entire range of the Péclet number $(P e)$, neglecting hydrodynamic interactions. Here, $P e$ is defined as the non-dimensional velocity of the probe. The microstructure is employed to calculate the average external force on the probe, from which one can infer a "microviscosity" of the dispersion via Stokes drag law. The microviscosity is computed as a function of the aspect ratio of the probe, $\hat{a}=a / b$, thereby delineating the role of the probe's shape. For a prolate probe, regardless of the value of $\hat{a}$, the microviscosity monotonically decreases, or "velocity thins," from a Newtonian plateau at small $P e$ until a second Newtonian plateau is reached as $P e \rightarrow \infty$. After appropriate scaling, we demonstrate this behavior to be in agreement with microrheology studies using spherical probes [Squires and Brady, "A simple paradigm for active and nonlinear microrheology," Phys. Fluids 17(7), 073101 (2005)] and conventional (macro-)rheological investigations [Bergenholtz et al., "The non-Newtonian rheology of dilute colloidal suspensions," J. Fluid. Mech. 456, 239-275 (2002)]. For an oblate probe, the microviscosity again transitions between two Newtonian plateaus: for $\hat{a}<3.52$ (to two decimal places) the microviscosity at small $P e$ is greater than at large $P e$ (again, velocity thinning); however, for $\hat{a}>3.52$ the microviscosity at small $P e$ is less than at large $P e$, which suggests it "velocity thickens" as $P e$ is increased. This anomalous velocity thickening - due entirely to the probe shape-highlights the care needed when designing microrheology experiments with nonspherical probes. () 2008 The Society of Rheology. [DOI: 10.1122/1.2821894]
\end{abstract}

\section{INTRODUCTION}

Over the past decade, a number of experimental techniques have emerged with the ability to infer rheological properties of complex fluids and biological materials at the micrometer scale. Collectively, they have come to be known as "microrheology" [for

\footnotetext{
a) Author to whom correspondence should be addressed. Present address: Department of Chemical Engineering, University of California Santa Barbara 93106-5080. Electronic mail: akhair@engineering.ucsb.edu
} 
reviews see MacKintosh and Schmidt (1999) and Waigh (2005)]. The name microrheology was adopted, perhaps, to distinguish these techniques from more traditional (macro)rheological procedures (e.g., mechanical rheometry), which typically operate on much larger (millimeter or more) length scales. Therein lies the main advantage of micro over macro rheology: It requires much smaller (microliter) amounts of sample. This is a particular advantage for rare, expensive, or biological substances that one simply cannot produce or procure in quantities sufficient for macrorheological testing.

At the heart of microrheology is the use of colloidal "probe" particles embedded in the material of interest. Through tracking the motion of the probe (using, e.g., light scattering, diffusing-wave spectroscopy, or laser-deflection particle tracking) one aims to infer the viscoelastic properties of the material. In passive tracking experiments the probe moves diffusively due to the random thermal fluctuations of its surrounding environment. The mean-squared displacement of the probe is measured, from which the complex, or frequency-dependent, shear modulus of the material is inferred via a generalized StokesEinstein-Sutherland relation [Mason and Weitz (1995); Mason et al. (1997)]. Many diverse systems, such as polymer gels [Mahaffy et al. (2000)], single cells [Daniels et al. (2006)], colloidal dispersions [Sohn and Rajagopalan (2004)], and actin networks [Gittes et al. (1997)], have been studied using passive microrheology. One should not think, however, that the use of thermally diffusing probes is limited to ascertaining viscoelastic moduli: recent studies have employed them to study protein folding [Tu and Breedveld (2005)], liquid-solid interfaces [Joly et al. (2006)], and vortices in non-Newtonian fluids [Atakhorrami et al. (2005)].

In active microrheology experiments, a material is disturbed by the externally forced motion of a probe particle. For example, Amblard et al. (1996) examined the viscoelasticity of Actin networks using colloidal probes driven by an external magnetic field. Furthermore, Hough and Ou-Yang (1999) inferred the frequency-dependent modulus of a polymer network from the small-amplitude oscillations (induced via a harmonically driven optical tweezer) of a silica probe. On a slightly different note, Holzwarth et al. (2002) have considered the transport of latex "cargo" probes in cytoplasm via the motor protein kinesin, as a model for intracellular transport of vesicles.

Through the application of a finite-amplitude forcing to a probe, active microrheology can been used to study material response beyond the small-amplitude, or linear-response, regime to which passive microrheology is limited. Recently, such nonlinear active microrheological techniques have been used to study colloids near the glass transition [Habdas et al. (2004)]; shear-thinning in colloidal dispersions [Meyer et al. (2006)]; and cluster formation in suspensions of rod-like particles [Wensink and Löwen (2006)].

Our own recent work [Squires and Brady (2005); Khair and Brady $(2005,2006)]$ has focused on developing theoretical paradigms for active-microrheology experiments in the nonlinear regime, by studying possibly the simplest of scenarios: an externally driven spherical probe in a monodisperse hard-sphere colloidal dispersion. The moving probe pushes the microstructure of the colloidal "bath" particles out of equilibrium. In turn, the progress of the probe is retarded by the presence of the bath particles, which, through Brownian diffusion, act to restore equilibrium. The relative magnitude of the probe's externally driven motion to the thermal restoring force of the bath particles sets the degree of microstructural distortion and is known as the Péclet number, $\mathrm{Pe}$.

One may interpret the retardation of the probe's motion in terms of an increase to the "microviscosity" of the dispersion (above the solvent viscosity) via application of Stokes drag law. However, as shown by Squires and Brady (2005), the computed value of the microviscosity depends on the whether the probe is driven at fixed force or fixed velocity, and on the probe to bath particle size ratio. Therefore, it should be clear that the micro- 
viscosity is not an intrinsic material property of the dispersion-it depends fundamentally on the nature of the probe particle itself. Nevertheless, after careful scaling, Squires and Brady (2005) demonstrate qualitative agreement between the microviscosity and the shear, or macro-, viscosity from macrorheology. Further work [Khair and Brady (2006)] investigated the role of hydrodynamic interactions between the probe and bath particles: in particular, when the particles experience short-range lubrication forces the microviscosity "force-thickens" at large $P e$, in analogy to the "shear thickening" of the macroviscosity [Bergenholtz et al. (2002)]. However, this near-quantitative agreement between micro- and macro-viscosity is only possible as the relevant scalings for both are known $a$ priori, allowing for a direct and meaningful comparison of the two. As noted by Squires and Brady (2005), for more complex (or unknown) materials, where this is not the case, one should not expect micro and macro to agree in general.

In the present work, we examine another facet of active microrheology; namely, what role does the shape of the probe play? In passive microrheology the issue of probe shape has been addressed by Cheng and Mason (2003), who studied the rotational diffusion of a wax microdisk in a polymer solution. By measuring the mean-squared angular displacement of the disk about its symmetry axis, they inferred the frequency-dependent shear modulus of the polymer solution via a rotational generalized Stokes-Einstein-Sutherland relation. Furthermore, Cheng et al. (2002) monitored the rotational fluctuations of a microdisk that is "orientationally trapped" via laser tweezers. Admittedly, the symmetry of a spherical probe greatly simplifies the design and analysis of microrheological experiments. Unfortunately, this symmetry can also be viewed as a drawback: One can infer only a scalar (micro-)viscosity from a (single) spherical probe, whereas in macrorheology the full stress tensor is obtainable, including normal stress differences and an isotropic osmotic pressure [Bergenholtz et al. (2002)]. Naturally, this leads one to ask if a nonspherical probe might give more than just a microviscosity. For example, could it be used to infer normal stress differences? In this (first) study, however, we shall ask the simpler question of how the shape of the probe affects the computed value of the microviscosity. Certainly, this is an issue of experimental significance as one expects the probe to have some degree of non-sphericity, as result of the manufacturing process or due to surface asperities, for instance.

As a variation of the model system used in our previous work, we consider a nonspherical probe translating at constant velocity through a dispersion of spherical bath particles. The probe is a body of revolution with major and minor semiaxes $a$ and $b$, respectively, and the bath particles are spheres of radii $b$. The probe's shape is such that when its major(minor) axis is the axis of revolution the excluded-volume, or contact, surface between the probe and a bath particle is a prolate(oblate) spheroid. ${ }^{1}$ To facilitate an analytical treatment we assume the volume fraction of bath particles is small compared to unity and neglect solvent-mediated hydrodynamic interactions between the probe and bath particles. In this limit, the pair-distribution function, which represents the likelihood of finding a bath particle at a particular location from the probe (and hence is a measure of the nonequilibrium microstructure), satisfies a two-body Smoluchowski equation. Our main focus in this paper is on a (prolate or oblate) probe moving along its

\footnotetext{
${ }^{1}$ The reader may wonder why we did not simply take the probe to be a spheroid (in both oblate and prolate cases). It is, of course, possible to do this, but the resulting excluded-volume shape is rather complicated: specifically, it is not a coordinate surface in spheroidal coordinates, making the ensuing analysis far more difficult. We have also limited the analysis to bath particles of radii equal to the minor semiaxes of the probe, $b$, in order that the probe-bath geometry is determined by a single parameter: the probe aspect ratio $\hat{a}=a / b$. For a discussion of some of the interesting situations that occur when this assumption is relaxed, see Sec. VII.
} 
symmetry axis, for which the pair-distribution function is axisymmetric about the direction of motion. From the pair-distribution function we calculate the average external force required to sustain the probe's motion, which can be interpreted in terms of a microviscosity via application of Stokes law. Our aim is to calculate the nonequilibrium microstructure and microviscosity over a wide range of probe aspect ratio $\hat{a}=a / b$ and $P e$ (here, $P e$ is the dimensionless velocity of the probe).

The rest of this paper is organized as follows. In Sec. II we present the Smoluchowski equation governing the nonequilibrium microstructure of the dispersion. Details of the coordinate systems used for modeling a prolate and oblate probe translating along its symmetry axis are given in Secs. II A and II B, respectively. In Sec. III we demonstrate how the average external force on the probe can be interpreted as a microviscosity of the dispersion. In Sec. IV we present a perturbation analysis of the Smoluchowski equation in the limits of small $(P e \ll 1)$ and large $(P e \gg 1)$ departures from equilibrium. For arbitrary $P e$ the Smoluchowski equation is solved numerically, and the techniques used for doing so are explained in Sec. V. Results for the microstructure and microviscosity are detailed in Sec. VI. In Sec. VII we offer some concluding remarks, including a preliminary analysis of a prolate probe translating at an angle $\beta$ to its symmetry axis. In this case, one must apply an external torque to prevent the probe from rotating, and we suggest how the torque may be indicative of the normal stress differences of the dispersion.

\section{NONEQUILIBRIUM MICROSTRUCTURE}

Consider a probe particle traveling at constant velocity through a dispersion of colloidal bath particles suspended in a Newtonian fluid of shear viscosity $\eta$. The probe is a body of revolution with major and minor semiaxes $a$ and $b$, respectively, and the bath particles are spheres of radii $b$. To develop an analytical description of the microstructural deformation caused by the driven probe, we assume the volume fraction of bath particles, $\phi=4 \pi n b^{3} / 3$ (with $n$ the number density of bath particles), is much less than unity. In this (dilute) limit the microstructure is determined by interactions between the probe and a single bath particle, and the pair-distribution function $g(\mathbf{r})$ [defined as $n g(\mathbf{r})=P_{1 / 1}(\mathbf{r})$, with $P_{1 / 1}(\mathbf{r})$ the conditional probability of finding a bath particle at a separation $\mathbf{r}$ from the probe] satisfies a two-body Smoluchowski equation, viz.

$$
D \nabla_{r}^{2} g+\mathbf{U} \cdot \nabla_{r} g=0
$$

where $D=k T / 6 \pi \eta b$ is the Stokes-Einstein-Sutherland diffusivity of a bath particle ( $k T$ being the thermal energy), and $\mathbf{U}$ is the probe velocity. [For a systematic derivation of Eq. (1), starting from the $N$-body Smoluchowski equation, see Squires and Brady (2005)]. In writing Eq. (1) the relative coordinate system $\mathbf{r}=\mathbf{x}_{2}-\mathbf{x}_{1}$ and $\mathbf{z}=\mathbf{x}_{2}+\mathbf{x}_{1}$ is employed, where $\mathbf{x}_{1}$ and $\mathbf{x}_{2}$ denote the probe and bath particle positions, respectively.

To non-dimensionalize the Smoluchowski equation we scale lengths with $b$ and velocities by $U=|\mathbf{U}|$, which gives

$$
\nabla^{2} g+P e \hat{\mathbf{U}} \cdot \nabla g=0
$$

where $\hat{\mathbf{U}}=\mathbf{U} / U$, and we have dropped the subscript $r$ for brevity. A Péclet number, Pe $=U b / D$, arises from the scaling and may be thought of as a ratio of advective $(U)$ to diffusive $(D / b)$ "velocities." Far from the probe, it is assumed that the dispersion has no long-range order:

$$
g(\mathbf{s}) \rightarrow 1 \quad \text { as }|\mathbf{s}| \rightarrow \infty,
$$

with $\mathbf{s}=\mathbf{r} / b$. The rigidity of the particles is represented by a no-flux condition: 


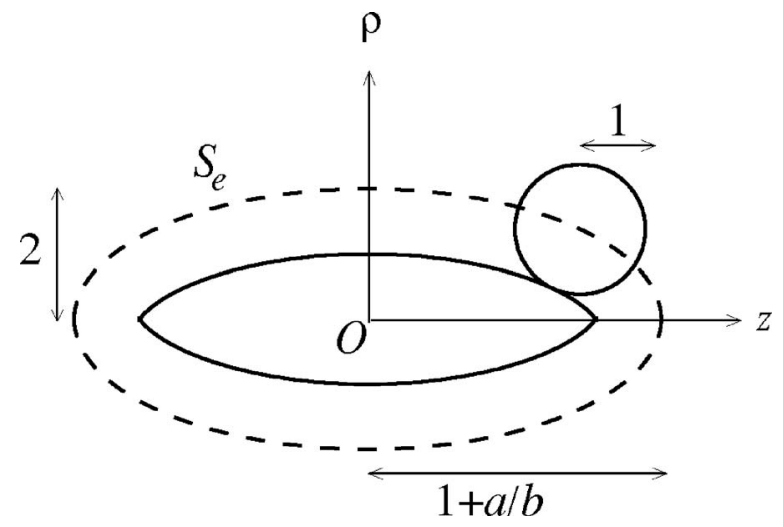

FIG. 1. Definition sketch for the prolate probe. Here, the aspect ratio $\hat{a}=a / b=3.5$. The circle of radius unity represents a bath particle, which is contacting the probe. The probe moves at constant velocity along its axis of revolution, the $z$ axis. The excluded-volume surface $S_{e}$ (broken curve) is formed by "rolling" the bath particle over the probe's surface.

$$
\mathbf{n} \cdot(\nabla g+P e \hat{\mathbf{U}} g)=0 \quad \text { on } S_{e},
$$

where $S_{e}$ is the excluded-volume, or contact, surface between the probe and bath particle. Clearly, the form of $S_{e}$ is dependent on the shape of the probe. In this study, we take the probe shape to be that which results in a prolate(oblate) spheroidal $S_{e}$ when the probe's axis of revolution is its major(minor) axis. In both cases, when the probe moves along its symmetry axis the microstructure is axisymmetric about the direction of motion; however, the two situations require different coordinate systems with which to solve the Smoluchowski equation, as discussed next.

\section{A. Prolate probe}

We consider the probe to move along its major axis, which is taken as the $z$ axis of a cylindrical $[\rho, z]$ coordinate system, whose origin is at the center of the probe (see Fig. 1). The probe's shape is such that the excluded-volume surface $S_{e}$ is a prolate spheroid with major and minor semiaxes $a_{e}=1+\hat{a}$ and $b_{e}=2$, respectively, where $\hat{a}=a / b$ is the aspect ratio of the probe. (Recall, all lengths are made dimensionless with the bath particle radius $b$.) Let us introduce the prolate spheroidal coordinates $\xi$ and $\eta$ defined by

$$
\rho=c \sinh \xi \sin \eta, \quad z=c \cosh \xi \cos \eta,
$$

where $c$ is a scale factor; $0 \leqslant \xi<\infty$; and $0 \leqslant \eta \leqslant \pi$. The coordinate surface $\xi=\xi_{0}$ is a prolate spheroid (centered at the origin) with major and minor semiaxes $a_{0}=c \cosh \xi_{0}$ and $b_{0}=c \sinh \xi_{0}$, respectively. Thus, we find

$$
c=\sqrt{(\hat{a}+1)^{2}-4}, \quad \xi_{e}=\frac{1}{2} \ln \left(\frac{\hat{a}+3}{\hat{a}-1}\right),
$$

with $\xi_{e}$ the coordinate surface corresponding to $S_{e}$. In cylindrical coordinates $S_{e}$ is represented as $\left[\rho_{e}=2 \sin \eta, z_{e}=(1+\hat{a}) \cos \eta\right]$, and the probe itself is 


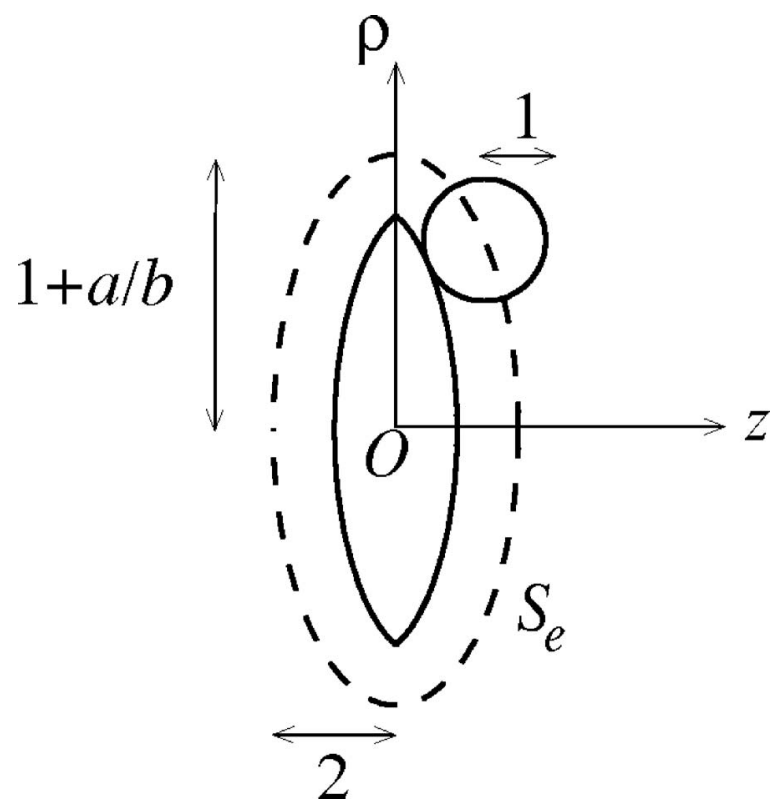

FIG. 2. Definition sketch for the oblate probe. Here, the aspect ratio $\hat{a}=a / b=3.5$, and the legend is the same as in Fig. 1. Again, the probe moves along its axis of revolution (the $z$ axis) at constant velocity.

$$
\left[\rho_{p}=\rho_{e}-\frac{\cosh \xi_{e} \sin \eta}{\left(\sinh ^{2} \xi_{e}+\sin ^{2} \eta\right)^{1 / 2}}, \quad z_{p}=z_{e}-\frac{\sinh \xi_{e} \sin \eta}{\left(\sinh ^{2} \xi_{e}+\sin ^{2} \eta\right)^{1 / 2}}\right] .
$$

Note, when $\hat{a}=1$ the probe and excluded-volume surfaces degenerate to spheres, of radii 1 and 2 , respectively.

In prolate spheroidal coordinates, the Smoluchowski equation (2) becomes

$$
\begin{aligned}
& \frac{1}{\sinh \xi} \frac{\partial}{\partial \xi}\left(\sinh \xi \frac{\partial g^{p r}}{\partial \xi}\right)+\frac{1}{\sin \eta} \frac{\partial}{\partial \eta}\left(\sin \eta \frac{\partial g^{p r}}{\partial \eta}\right) \\
& =c P e\left(\cosh \xi \sin \eta \frac{\partial g^{p r}}{\partial \eta}-\sinh \xi \cos \eta \frac{\partial g}{\partial \xi}\right),
\end{aligned}
$$

subject to the boundary conditions

$$
\begin{gathered}
\frac{\partial g^{p r}}{\partial \xi}=-c P e \sinh \xi_{e} \cos \eta g^{p r} \quad \text { at } \xi=\xi_{e} \\
g^{p r} \rightarrow 1 \quad \text { as } \xi \rightarrow \infty
\end{gathered}
$$

The superscript $p r$ has been added to the pair-distribution function to make clear these are equations are for the prolate probe. Equations pertaining to the oblate probe will have attached the superscript $o b$.

\section{B. Oblate probe}

In this case, the probe moves along its minor axis, which is the $z$ axis of a cylindrical $[\rho, z]$ coordinate system. The shape of $S_{e}$ is an oblate spheroid, with the same major and minor semiaxes as in the prolate case (see Fig. 2). We define the oblate spheroidal 
coordinates $\xi$ and $\eta$ by

$$
\rho=c \cosh \xi \sin \eta, \quad z=c \sinh \xi \cos \eta,
$$

where $\xi$ and $\eta$ have the same ranges as before; moreover, the scale factor $c$ and the excluded-volume coordinate surface $\xi_{e}$ are again given by Eq. (5). In fact, one can transform from prolate to oblate coordinates via the transformation

$$
c \rightarrow-i c, \quad \cosh \xi \rightarrow i \sinh \xi,
$$

where $i=\sqrt{-1}$ [Morse and Feshbach (1953)]. Therefore, in the interests of brevity, rather than state explicitly the excluded-volume shape, probe shape, and Smoluchowski equation in oblate spheroidal coordinates, we note that they can be obtained by applying Eq. (8) to the relevant prolate spheroidal equations. Furthermore, in the what follows we perform detailed calculations for the prolate probe and, wherever possible, employ Eq. (8) to obtain results for the equivalent (i.e., of equal aspect ratio, $\hat{a}$ ) oblate probe.

\section{MICROVISCOSITY}

From the pair-distribution function it is possible to calculate many microstructurally averaged properties. In the present context, the most interesting is the average external force on the probe $\left\langle\mathbf{F}^{e x t}\right\rangle$, which, as derived by Squires and Brady (2005), is given by

$$
\left\langle\mathbf{F}^{e x t}\right\rangle=M^{-1} \mathbf{U}+n k T \oint \mathbf{n} g(\mathbf{r}) d S_{e},
$$

where $M=1 / 6 \pi \eta b K$ is the mobility of the probe, and $d S_{e}$ is the differential area element of the excluded-volume surface. The mobility factor $K$ accounts for the non-sphericity of the probe. For the rather complicated probe shape represented by Eq. (6) evaluation of $K$ is nontrivial. Therefore, we make the assumption that (for purposes of computing the mobility only) the probe itself may be approximated as a spheroid with the same major and minor semiaxes. Thus, the mobility factors are [Happel and Brenner (1965)]

$$
\begin{aligned}
K^{p r} & =\frac{4}{3}\left(\tau_{p}^{2}-1\right)^{-1 / 2}\left[\left(\tau_{p}^{2}+1\right) \operatorname{coth}^{-1} \tau_{p}-\tau_{p}\right]^{-1}, \\
K^{o b} & =\frac{4 \hat{a}}{3}\left(\lambda_{p}^{2}+1\right)^{-1 / 2}\left[\lambda_{p}-\left(\lambda_{p}^{2}-1\right) \cot ^{-1} \lambda_{p}\right]^{-1},
\end{aligned}
$$

where $\tau_{p}=\cosh \xi_{p}$ and $\lambda_{p}=\sinh \xi_{p}$, and

$$
\xi_{p}=\frac{1}{2} \ln \left(\frac{\hat{a}+1}{\hat{a}-1}\right)
$$

is the coordinate surface of the "spheroidal" probe. In Fig. 3 we plot $K^{p r}$ and $K^{o b}$ as a function of the aspect ratio $\hat{a}=a / b$. In the limit of a spherical probe, $\hat{a}=1$, both $K^{p r}$ and $K^{o b}$ approach unity, as expected. When $\hat{a} \gg 1$ the prolate probe resembles a long thin rod and $K^{p r} \sim 2 \hat{a} /[3 \ln (2 \hat{a})-3 / 2]$ [cf. Eq. (4-31.4) of Happel and Brenner (1965)]. The oblate probe in the limit $\hat{a} \gg 1$ degenerates to a thin circular disk, for which $K^{o b} \sim 8 \hat{a} / 3 \pi$ [cf. Eq. (4-27.2) of Happel and Brenner (1965)].

As the probe translates, there is an accumulation(deficit) of bath particles on its upstream(downstream) side, resulting in an "osmotic pressure" imbalance [the integral in Eq. (9) is simply the average of this imbalance over the excluded-volume surface], which provides an entropic force resisting the probe's motion. As such, the average external 


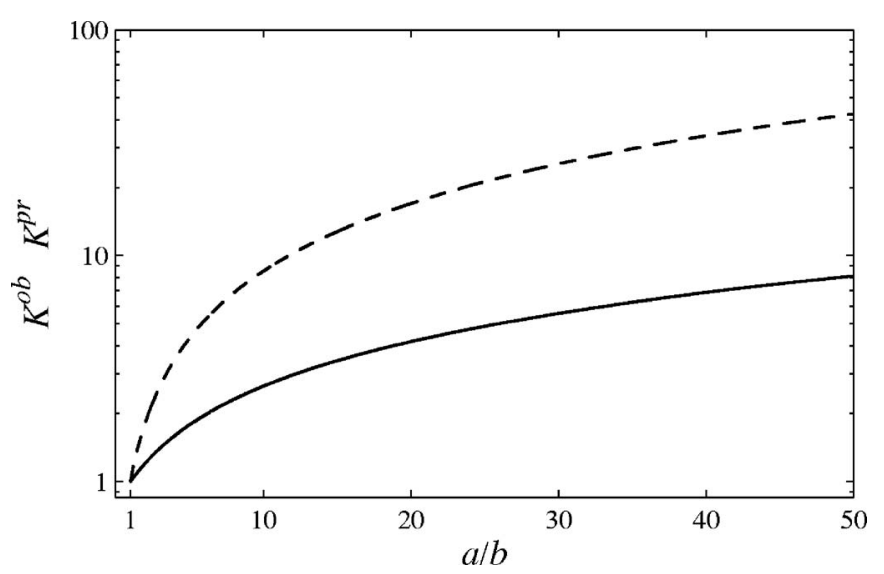

FIG. 3. Plot of the mobility factors versus probe aspect ratio $\hat{a}=a / b$. The broken line is the oblate factor $K^{o b}$ and the solid line is the prolate factor $K^{p r}$.

force on the probe required to maintain its velocity at $\mathbf{U}$ is greater than if the probe were traveling in the absence of bath particles. One can interpret the increase in external force as an increment to a "microviscosity" of the dispersion: from Stokes drag law, $\left\langle F_{z}^{\text {ext }}\right\rangle$ $=6 \pi \eta b K U \eta_{r}$ (the external force is, by symmetry, directed solely along the $z$-axis), a dimensionless relative microviscosity $\eta_{r}$ is defined

$$
\eta_{r}=1+\frac{n k T}{6 \pi \eta b K U} \oint n_{z} g d S_{e},
$$

where $n_{z}=\mathbf{n} \cdot \hat{\mathbf{U}}$. It is natural to define a dimensionless relative microviscosity increment, $\Delta \eta_{r}$, as

$$
\Delta \eta_{r}=\frac{n k T}{6 \pi \eta b K U} \oint n_{z} g d S_{e},
$$

representing the contribution to the microviscosity due to the interaction of the probe with bath particles. For the prolate probe the increment may be written as

$$
\Delta \eta_{r}^{p r}=\frac{6 \phi}{K^{p r} P e} \int_{0}^{\pi} g^{p r} \cos \eta \sin \eta d \eta .
$$

Recall, $\phi=4 \pi n b^{3} / 3$ is the volume fraction of bath particles. In the oblate case the increment is

$$
\Delta \eta_{r}^{o b}=\frac{3(1+\hat{a})^{2} \phi}{2 K^{o b} P e} \int_{0}^{\pi} g^{o b} \cos \eta \sin \eta d \eta
$$

\section{ANALYTICAL RESULTS}

\section{A. Near equilibrium $P e \ll 1$}

For small $\mathrm{Pe}$ the Brownian diffusion of bath particles dominates over advection by the moving probe; the microstructure is only slightly perturbed from its equilibrium state. This is the passive-microrheology, or linear-response, regime in which the microstructural deformation is proportional to the probe velocity. Therefore, we write the pair- 


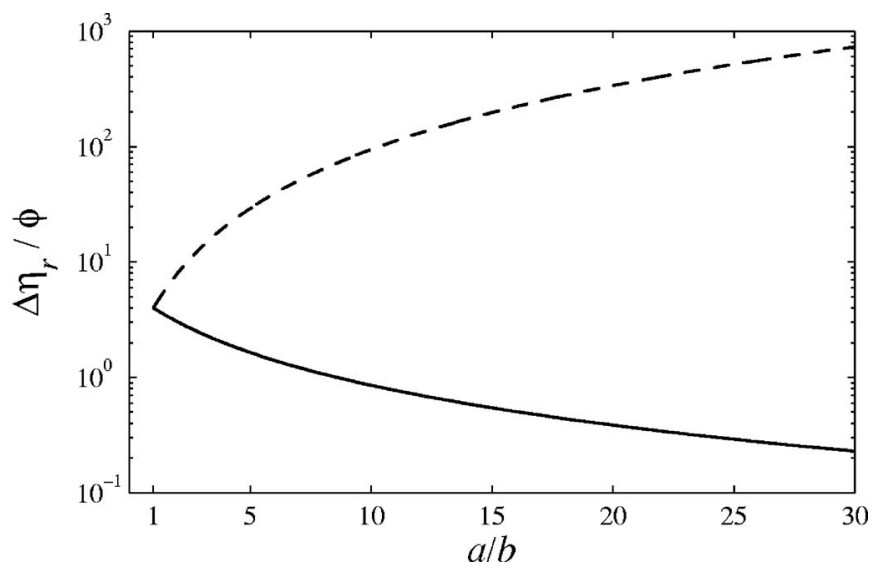

FIG. 4. Microviscosity increments at small $P e$ as a function of probe's aspect ratio $\hat{a}=a / b$. The broken line is the oblate increment $\Delta \eta_{r}^{o b}$ and the solid line is the prolate increment $\Delta \eta_{r}^{p r}$.

distribution function as $g=1+P e f$, where $f$ satisfies Laplace's equation and must vanish at large distances. In the prolate case the no-flux condition on the excluded-volume surface reads

$$
\frac{\partial f^{p r}}{\partial \xi}=-c \sinh \xi_{e} \cos \eta \text { at } \xi=\xi_{e}
$$

representing a dipolar forcing. The solution for $f^{p r}$ is found to be

$$
f^{p r}=\frac{c\left(\tau_{e}^{2}-1\right)}{\operatorname{coth}^{-1} \tau_{e}-\tau_{e}\left(\tau_{e} \operatorname{coth}^{-1} \tau_{e}-1\right)}\left(\tau \operatorname{coth}^{-1} \tau-1\right) \cos \eta,
$$

where $\tau=\cosh \xi$ and $\tau_{e}=\cosh \xi_{e}$. We note the "angular" dependence of $\cos \eta$, giving $f^{p r}$ the structure a diffusive dipole directed along $\hat{\mathbf{U}}$. From Eq. (11), the microviscosity increment is calculated as

$$
\Delta \eta_{r}^{p r}=\frac{1}{K^{p r}}\left[\frac{8\left(\tau_{e}^{2}-1\right)^{1 / 2}\left(\tau_{e} \operatorname{coth}^{-1} \tau_{e}-1\right)}{\operatorname{coth}^{-1} \tau_{e}-\tau_{e}\left(\tau_{e} \operatorname{coth}^{-1} \tau_{e}-1\right)}\right] \phi,
$$

where we have used $c\left(\tau_{e}^{2}-1\right)^{1 / 2}=2$. Using the transformation (8) on Eq. (13), we find in the oblate case

$$
f^{o b}=\frac{c\left(\lambda_{e}^{2}+1\right)}{\lambda_{e}\left(1-\lambda_{e} \cot ^{-1} \lambda_{e}\right)-\cot ^{-1} \lambda_{e}}\left(\lambda \cot ^{-1} \lambda-1\right) \cos \eta,
$$

where $\lambda=\sinh \xi$ and $\lambda_{e}=\sinh \xi_{e}$. From Eq. (12) the microviscosity increment is calculated as

$$
\Delta \eta_{r}^{o b}=\frac{(1+\hat{a})^{3}}{K^{o b}}\left[\frac{\left(1+\lambda_{e}^{2}\right)^{1 / 2}\left(\lambda_{e} \cot ^{-1} \lambda_{e}-1\right)}{\lambda_{e}\left(1-\lambda_{e} \cot ^{-1} \lambda_{e}\right)-\cot ^{-1} \lambda_{e}}\right] \phi,
$$

where we have used $c\left(\lambda_{e}^{2}+1\right)^{1 / 2}=\hat{a}+1$.

Figure 4 plots the prolate $\left(\Delta \eta_{r}^{p r}\right)$ and oblate $\left(\Delta \eta_{r}^{o b}\right)$ microviscosity increments at small $P e$ as a function of the aspect ratio $\hat{a}=a / b$. For $\hat{a}=1$ both increments approach the value $4 \phi$, which is the microviscosity increment for a spherical probe at small $\mathrm{Pe}$ [Squires and Brady (2005)]. On increasing the aspect ratio the prolate(oblate) increment decays- 
(grows) monotonically and vanishes(diverges) in the limit $\hat{a} \rightarrow \infty$. We defer a physical explanation of these trends until presentation of our numerical computations at arbitrary $P e$ (Sec. VI).

\section{B. Far from equilibrium $P e \gg 1$}

For large $P e$ advection dominates the microstructure, and Brownian diffusion is important only in a thin boundary layer on the upstream side of the probe. The boundary layer represents (in a frame fixed on the moving probe) a balance between the strong advection of bath particles (with velocity $-\hat{\mathbf{U}}$ ) towards the probe and diffusion, which enables the bath particles to pass around the (impenetrable) probe. As a result, there is a large accumulation of bath particles $(g \gg 1)$ in the boundary layer. Downstream of the probe advection carries bath particles away: A region of low-particle density $(g \approx 0)$, or wake, is present. In this subsection, we present a simple physical argument to calculate the boundary-layer microstructure, which is based on the analysis of Squires and Brady (2005) for the microstructure around a spherical probe at large Pe. However, note that (as shown in Appendix B) the same conclusions may be obtained from a rigorous asymptotic analysis. In what follows, we examine the oblate and prolate cases separately.

\section{Oblate probe}

As mentioned above, the boundary layer signifies a balance between "radial" diffusion $\left(D \nabla_{\xi}^{2} g\right)$ and advection $\left(\mathbf{U}_{\perp} \cdot \nabla_{\xi} g\right)$ with the perpendicular component of the probe velocity $\left(U_{\perp}=\mathbf{U} \cdot \hat{\boldsymbol{\xi}}\right)$. Equating these two terms gives an approximate (first order) model for the microstructure in the boundary layer, which, in dimensional terms, reads

$$
D \frac{\partial^{2} g^{o b}}{\partial t^{2}}+U c \cosh \xi_{e} \cos \eta \frac{\partial g^{o b}}{\partial t}=0
$$

where $t=\xi-\xi_{e}$ is a dimensionless coordinate perpendicular to the (local) excludedvolume surface $\left(S_{e}\right)$, and $c \cosh \xi_{e}=a+b$ is the major semiaxes of $S_{e}$. The solution of Eq. (14) is

$$
g^{o b} \sim A^{o b}(\eta) e^{-U(a+b) t \cos \eta / D} .
$$

Clearly, this solution is valid only upstream of the probe, $0 \leqslant \eta \leqslant \pi / 2$; downstream there is a wake in which $g \approx 0$. We integrate across the boundary layer to obtain the local surface density $\sigma$ :

$$
\sigma=\int_{0}^{\infty} A^{o b}(\eta) e^{-U(a+b) t \cos \eta / D} d t=\frac{D A^{o b}(\eta)}{U(a+b) \cos \eta}
$$

At steady state $\sigma$ must be independent of the angular coordinate $\eta$, to ensure there is no accumulation of bath particles on $S_{e}$; thus, $A^{o b} \sim \cos \eta$. The constant of proportionality can be determined through a flux balance on the boundary layer. In general, the oblate shape of the probe leads to a complicated boundary-layer geometry. However, we can make progress in the limit $a \gg b$, where $S_{e}$ degenerates to a circular disk of radius $a+b$ and thickness $4 b$ (see Fig. 5). The flux of bath particles $Q_{\text {in }}$ towards the disk is simply

$$
Q_{\text {in }}=\pi(a+b)^{2} g^{\infty} U,
$$

where $g^{\infty}(=1)$ is the far-field value of the pair-distribution function. At steady state, $Q_{\text {in }}$ is balanced by a flux $Q_{\text {out }}$ of particles exiting the boundary layer. The bath-particle surface density $\sigma$ is advected around $S_{e}$ with the parallel component of velocity $U_{\|}=\mathbf{U} \cdot \hat{\boldsymbol{\eta}}=$ 


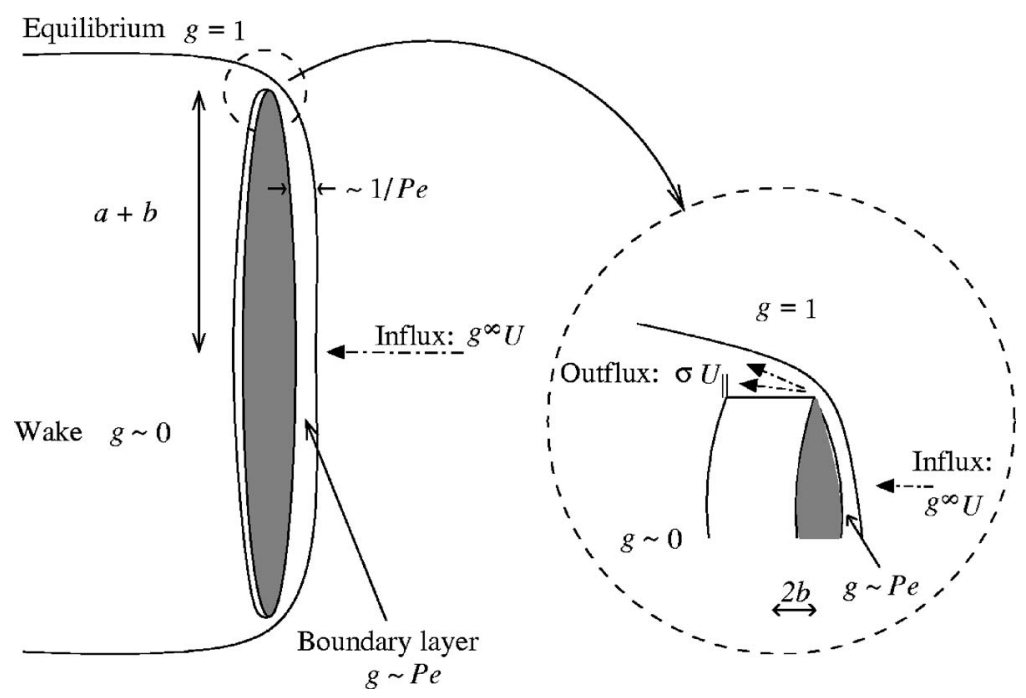

FIG. 5. Sketch of the microstructure around an oblate probe at large $P e$.

$-U \sinh \xi_{e} \sin \eta /\left(\cosh ^{2} \xi_{e}-\sin ^{2} \eta\right)^{1 / 2}$ and finally leaves the boundary layer from the edge of the disk, over a cylindrical (exit) surface of perimeter $2 \pi(a+b)$ and thickness $2 b$. (The thickness of the disk edge is $4 b$; however, the boundary layer terminates halfway along the edge. Thus, the thickness of the exit surface is $2 b$.) The edge corresponds to $\eta$ $=1 / 2$, for which $U_{\|} \approx-U$. Therefore, $Q_{\text {out }}$ is

$$
Q_{\text {out }}=4 \pi(a+b) b \sigma U \text {. }
$$

Equating fluxes gives

$$
\sigma=\frac{1}{4}\left(\frac{a+b}{b}\right) g^{\infty} .
$$

From Eqs. (15)-(17) we find the boundary layer microstructure to be (in dimensionless variables)

$$
g^{o b}(\xi, \eta<\pi / 2) \sim \frac{(\hat{a}+1)^{2}}{4} P e \cos \eta e^{-(\hat{a}+1) P e\left(\xi-\xi_{e}\right) \cos \eta} .
$$

The pair-distribution function at contact is

$$
g^{o b}\left(\xi_{e}, \eta<\pi / 2\right)=\frac{(\hat{a}+1)^{2}}{4} P e \cos \eta ;
$$

thus, there is a large $O(P e=U b / D)$ excess of bath particles in front of the probe. Moreover, this excess is amplified by a geometrical factor of $(\hat{a}+1)^{2} / 4$. Properly, though, for the circular disk (and, actually, for all oblate probes) one should scale lengths with the disk radius $a+b$ (it being the dominant length scale in the problem), which yields

$$
g^{o b}\left(\xi_{e}, \eta<\pi / 2\right)=\frac{(\hat{a}+1)}{4} P e_{a} \cos \eta,
$$

where $P e_{a}=U(a+b) / D$ is a Péclet number based on the major semiaxes of $S_{e}$, rather than the bath particle radius $b$. Now we find the $O\left(P e_{a}\right)$ buildup of bath particles is amplified 


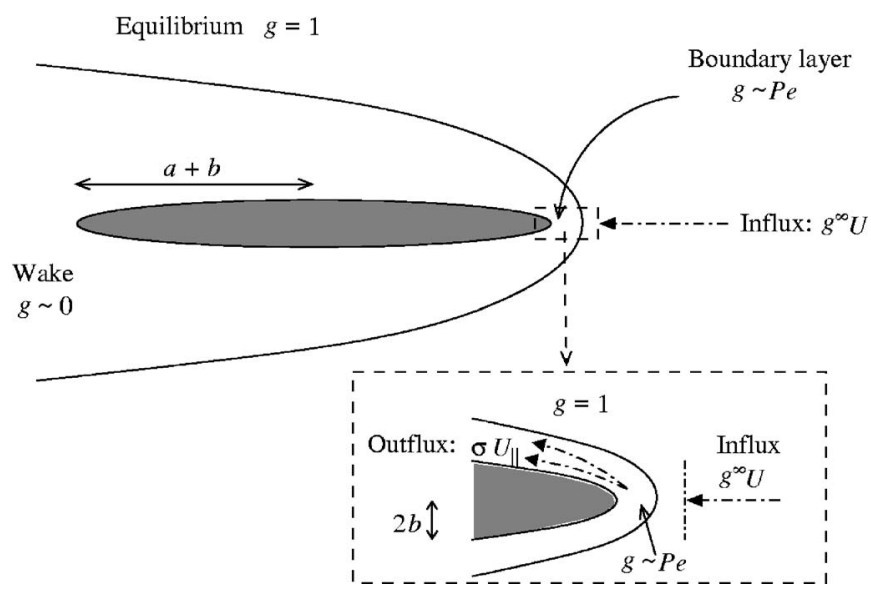

FIG. 6. Sketch of the microstructure around an prolate probe at large $P e$.

by $(\hat{a}+1) / 4$, which is simply the ratio of the surface areas for entry $\left[\pi(a+b)^{2}\right]$ and exit $[4 \pi b(a+b)]$ of bath particles: physically, bath particles are advected into the boundary layer through a much $[O(a+b)]$ larger area (the disk face) than from which they can escape (the disk edge), leading to large amplification of the $O(P e)$ buildup.

Finally, from Eqs. (12) and (18) (i.e., with the Péclet number based on the bath particle radius) the microviscosity increment is

$$
\Delta \eta_{r}^{o b}=\frac{3(1+\hat{a})^{2} \phi}{2 K^{o b} P e} \int_{0}^{\pi / 2} g^{o b} \cos \eta \sin \eta d \eta=\frac{(\hat{a}+1)^{4}}{8} \frac{\phi}{K^{o b}} .
$$

\section{Prolate probe}

For the prolate probe at large $P e$ an equation for the boundary-layer microstructure may be obtained by balancing again radial diffusion $\left(D \nabla_{\xi}^{2} g\right)$ and advection $\left(\mathbf{U}_{\perp} \cdot \nabla_{\xi} g\right)$, viz.

$$
D \frac{\partial^{2} g^{p r}}{\partial t^{2}}+U c \sinh \xi_{e} \cos \eta \frac{\partial g^{p r}}{\partial t}=0
$$

where $t=\xi-\xi_{e}$, and $c \sinh \xi_{e}=2 b$ is the minor semiaxes of the excluded-volume surface. The solution to Eq. (19) is

$$
g^{p r} \sim A^{p r}(\eta) e^{-2 U b t \cos \eta / D} .
$$

Integrating across the boundary layer we find the surface density $\sigma$ to be

$$
\sigma=\int_{0}^{\infty} A^{p r}(\eta) e^{-2 U b t \cos \eta / D} d t=\frac{D A^{p r}(\eta)}{2 U b \cos \eta} .
$$

As for oblate probe, the function $A^{p r}(\eta)$ can be found through a flux balance. To make progress, we consider the limiting case $a \gg b$, where the excluded volume degenerates to a long thin rod [of length $2(a+b)$ and diameter $4 b$ ]. A sketch of this configuration is shown in Fig. 6. The flux of bath particles $Q_{i n}$ entering the boundary layer (located at the upstream tip of the rod) is 


$$
Q_{\text {in }}=\pi(2 b)^{2} g^{\infty} U
$$

The surface density is advected past the tip of the rod with the parallel component of velocity $U_{\|}=\mathbf{U} \cdot \hat{\boldsymbol{\eta}}=-U \cosh \xi_{e} \sin \eta /\left(\sinh ^{2} \xi_{e}+\sin ^{2} \eta\right)^{1 / 2}$ and leaves the boundary layer along the length of the rod, over a cylindrical (exit) surface of perimeter $4 \pi b$ and length $a+b$. (The boundary layer terminates at the midpoint of the rod; hence the length of the exit surface is half that of the rod.) The long thin rod is obtained in the limit $\xi_{e} \rightarrow 0$, in which case $U_{\|} \approx-U$. Therefore, $Q_{\text {out }}$ is

$$
Q_{\text {out }}=4 \pi b(a+b) \sigma U .
$$

At steady state, setting $Q_{\text {in }}=Q_{\text {out }}$ gives

$$
\sigma=\left(\frac{b}{a+b}\right) g^{\infty}
$$

From Eqs. (20)-(22) the boundary layer microstructure is (in dimensionless variables)

$$
g^{p r}(\xi, \eta<\pi / 2) \sim \frac{2}{\hat{a}+1} P e \cos \eta e^{-2 P e\left(\xi-\xi_{e}\right) \cos \eta}
$$

and the contact value is

$$
g^{p r}\left(\xi_{e}, \eta<\pi / 2\right)=\frac{2}{\hat{a}+1} P e \cos \eta .
$$

As before, there is a large $O(P e=U b / D)$ excess of bath particle in front of the probe. However, in contrast to the oblate probe, the excess is attenuated by a factor of $2 /(\hat{a}$ $+1)$. Physically, bath particles exit the boundary layer through a much $[O(a+b)]$ larger area (the rod length) than from which they enter (the rod tip), leading to an attenuation of the $O(P e)$ buildup. Again, the attenuation factor is given (modulo a factor of 2 that could, actually, be incorporated into a new Péclet number based on the minor semiaxes of $\left.S_{e}: P e_{b}=2 b U / D\right)$ by the ratio of the surface areas for entry $\left[\pi(2 b)^{2}\right]$ and exit $[4 \pi b(a$ $+b)]$ of bath particles.

Last, from Eqs. (11) and (23) the microviscosity increment is

$$
\Delta \eta_{r}^{p r}=\frac{6 \phi}{K^{p r} P e} \int_{0}^{\pi / 2} g^{p r} \cos \eta \sin \eta d \eta=\frac{4}{\hat{a}+1} \frac{\phi}{K^{p r}} .
$$

Although our simple physical arguments are restricted to the limiting case $\hat{a}=a / b$ $\gg 1$, for which the prolate(oblate) excluded volume degenerates to a long thin rod(thin disk), the results [Eqs. (18) and (23)] are in fact valid for all $\hat{a}$ (see Appendix B). In Fig. 7 we plot the microviscosity increments at large $P e$ as a function of the aspect ratio $\hat{a}$ $=a / b$. For $\hat{a} \rightarrow 1$ both increments approach the value $2 \phi$, which is the microviscosity increment for a spherical probe at large $P e$ [Squires and Brady (2005)]. On increasing $\hat{a}$ the prolate(oblate) increment decays(grows) monotonically and vanishes(diverges) in the limit $\hat{a} \rightarrow \infty$.

Having determined the asymptotic values of the microviscosity for all $\hat{a}$, we now ask how it transitions between these limits. To this end, it is useful to examine the difference between the microviscosity in the limits $P e \ll 1$ and $P e \gg 1$ (see Fig. 8). For the prolate probe the microviscosity (for a fixed $\hat{a}$ ) at small $P e$ is greater than at large $P e$, which suggests the microviscosity decreases, or "velocity thins," as a function of Pe. However, the situation is not so simple for the oblate probe. On increasing $\hat{a}$ (above unity) the difference between small $P e$ and large $P e$ microviscosities is positive and growing, indi- 


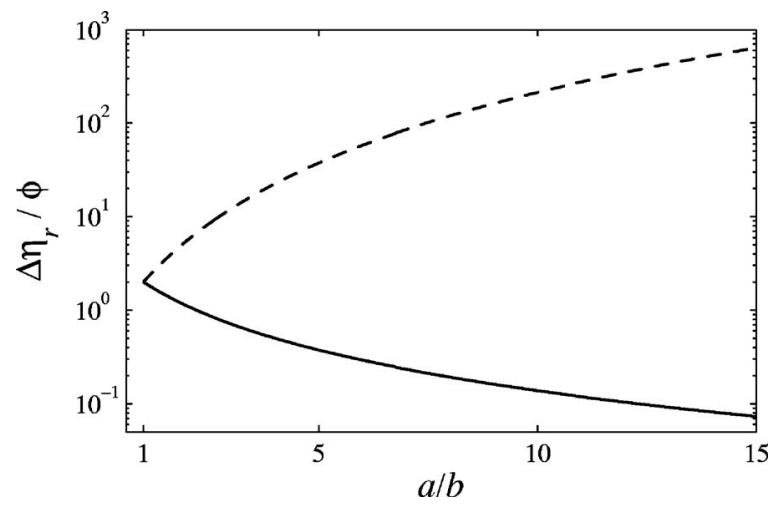

FIG. 7. Microviscosity increments at large $P e$ as a function of probe's aspect ratio $\hat{a}=a / b$. The legend is the same as in Fig. 4.

cating that the microviscosity velocity thins. This trend continues until $\hat{a}=1.89$ (to two decimal places), at which point the difference reaches a maximum value and proceeds to decrease monotonically with increasing $\hat{a}$. The difference in microviscosities is zero for $\hat{a}=3.52$ (to two decimal places), and for higher $\hat{a}$ it becomes increasingly negative. This behavior is rather unexpected and suggests that the microviscosity increment for the oblate probe increases, or "velocity thickens," as a function of $P e$ for $\hat{a}>3.52$. The conclusions of our asymptotic analysis will be verified by results from numerical solution of the Smoluchowski equation and a physical explanation for this unexpected behavior will be offered. Next, we describe the methods used to perform the numerical computations.

\section{NUMERICAL METHODS}

In the preceding section, we calculated the microstructure and microviscosity increments for small and large departures from equilibrium, using analytical perturbation methods. Here, we present numerical methods that facilitate computation of the microstructure and microviscosity at arbitrary $\mathrm{Pe}$. One should note, however, that the Smolu-

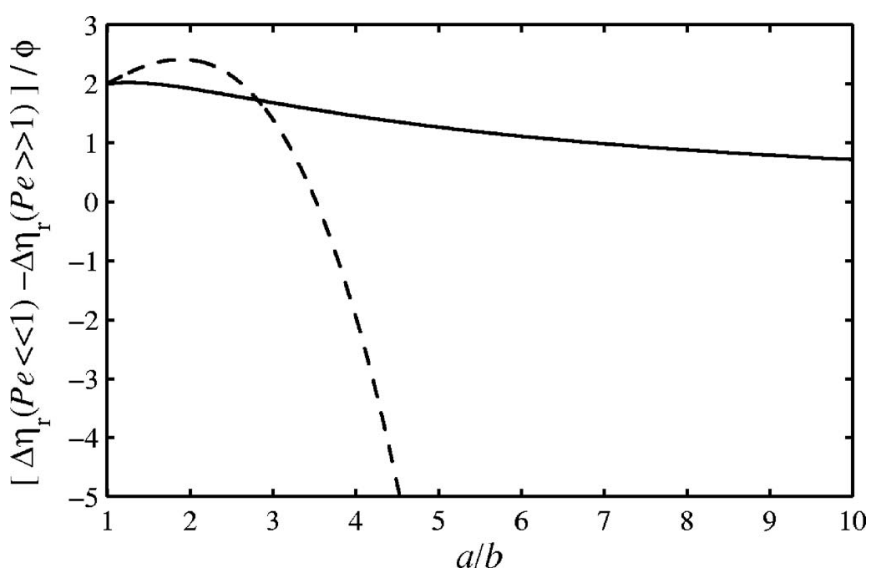

FIG. 8. Difference in microviscosity increments at small $P e$ and large $P e$ as a function of probe aspect ratio $\hat{a}=a / b$. The broken(solid) line is the difference in the oblate(prolate) increment 
chowski equation does in fact admit an exact solution in spheroidal coordinates (see Appendix A for details). Therefore, one could, in principle, use the exact solution to develop analytical formulas for the microviscosity valid for all $\mathrm{Pe}$. The exact solution is found by separation of variables and takes the usual form of a eigenfunction summation; unfortunately, the complexity of the eigenfunctions coupled with the mixed (Robin) no-flux boundary condition (4) on $S_{e}$, makes numerical evaluation of the exact solution computationally prohibitive (especially at large $P e$, where one needs to retain a large number of eigenfunctions). Therefore, we choose to solve the Smoluchowski equation numerically, using two different methods.

For $P e<10$ an expansion of the pair-distribution function in a series of Legendre polynomials is employed. This approach was used previously in determining the microstructure around an externally forced spherical probe [Khair and Brady (2006)]. As Pe increases one requires more terms in the expansion to faithfully represent the increasingly complex microstructure, which is computationally taxing. Therefore, for $P e>10$ we solve the Smoluchowski equation using a finite-difference scheme, which accurately captures the boundary layer and wake structure present at large $P e$. The two methods are described next.

\section{A. Legendre polynomial expansion}

For for the prolate probe, the pair-distribution $g^{p r}$ function is written as

$$
g^{p r}(\xi, \eta)=1+\sum_{n=0}^{\infty} f_{n}^{p r}(\xi) P_{n}(\cos \eta)
$$

where $P_{n}(z)$ is the Legendre polynomial of degree $n$ and argument $z$. This expansion is substituted into the Smoluchowski equation (7) and, after using the orthogonality property of the Legendre polynomials on the interval $[0, \pi]$, we arrive at a coupled set of ordinary differential equations for the expansion coefficients $f_{n}^{p r}(\xi)$ :

$$
\frac{1}{\sinh \xi}\left(\sinh \xi \frac{\partial f_{n}^{p r}}{\partial \xi}\right)-n(n+1) f_{n}^{p r}=-c P e\left(\alpha_{n} \cosh \xi+\beta_{n} \sinh \xi\right),
$$

where the advective coupling terms are

$$
\begin{gathered}
\alpha_{n}=\frac{(n+1)(n+2)}{2 n+3} f_{n+1}^{p r}+\frac{n(1-n)}{2 n-1} f_{n-1}^{p r}, \\
\beta_{n}=\frac{n+1}{2 n+3} \frac{\partial f_{n+1}^{p r}}{\partial \xi}+\frac{n}{2 n-1} \frac{\partial f_{n-1}^{p r}}{\partial \xi} .
\end{gathered}
$$

The boundary conditions on the expansion coefficients are

$$
\begin{gathered}
\frac{\partial f_{n}^{p r}}{\partial \xi}=-c \sinh \xi_{e} P e\left(\delta_{n 1}+\frac{n+1}{2 n+3} f_{n+1}^{p r}+\frac{n}{2 n-1} f_{n-1}^{p r}\right) \quad \text { at } \xi=\xi_{e} \\
f_{n}^{p r} \rightarrow 0 \quad \text { as } \xi \rightarrow \infty
\end{gathered}
$$

where $\delta_{i j}$ is Kronecker's delta. The system of equations is solved using the MATLAB boundary-value problem solver bvp $4 c$. The summation in Eq. (24) is truncated at $n$ $=n_{\max }$; for a given value of $P e$, the choice of $n_{\max }$ is made be requiring the resulting microviscosity increment not differ by more than $0.2 \%$ when computed using $n_{\max }$ and $n_{\max }+1$ terms. Of course, as $P e$ increases so does $n_{\max }$, which reflects the need for more 


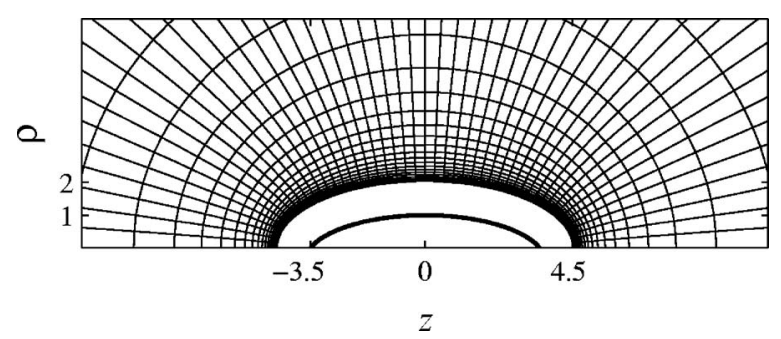

FIG. 9. Sample finite difference grid for a prolate probe. Here, $\hat{a}=3.5$; there are $50 \times 50$ grid points; and $P e$ $=5$. Note the high density of mesh points near the excluded-volume surface.

terms in the expansion to capture the microstructure. The largest value for which we obtained a solution was $P e=10$, requiring $n_{\max }=50$.

For the oblate probe the pair-distribution function $\left(g^{o b}\right)$ is expanded as per Eq. (24), and the differential equations for the expansion coefficients $\left(f_{n}^{o b}\right.$, say) can be derived by applying the transformation (8) to Eqs. (25) through (27).

\section{B. Finite differences}

Solving the Smoluchowski equation for $P e>1$ is numerically challenging: One has to capture the intricate boundary-layer structure at the front of the probe and the growing wake behind it. For $P e>10$ we approximate the Smoluchowski equation by a finitedifference equation (central differences are used for all derivatives) on a mesh that has a high density of grid points near the excluded-volume surface $S_{e}$ (to resolve the large gradients of the pair-distribution function in the boundary layer) and a lower density in the far field. To create this non-uniform mesh in (physical) $\xi$ space we employ a mapping from the semi-infinite domain $\xi \in\left[\xi_{e}, \infty\right)$ to the finite interval $p \in[0,1]$, viz.

$$
\ln p=-y\left(\omega+\frac{1-\omega}{1+y}\right),
$$

where $\omega$ is a parameter and $y=P e\left(\xi-\xi_{e}\right)$. Using a uniform grid in $p$ space one can (via adjustment of $\omega$ ) place a large number of nodes near $\xi=\xi_{e}$, to accurately model the near-field microstructure (e.g., see Fig. 9).

The linear system of equations resulting from discretization of the Smoluchowski equation is solved using a simple Jacobi iteration [the method closely resembles that of Khair and Brady (2006), who calculated the microstructure around a spherical probe at large $P e$ ]. As $P e$ increases, one requires a greater number of grid points for the method to converge: the maximum value of $P e$ for which a solution was obtained being $P e=100$.

\section{RESULTS}

In this section, we present results for the microstructure and microviscosity at arbitrary $P e$ for different values of $\hat{a}$. In Fig. 10 the microstructural deformation, $g-1$, is plotted in the symmetry plane of a prolate probe of aspect ratio $\hat{a}=3.5$, as a function of $P e$. For $P e \ll 1$ diffusion dominates: The deformation is approximately for-aft symmetric about the probe, with an $O(P e)$ accumulation(depletion) on its upstream(downstream) side, in agreement with the linear-response analysis of Sec. IV A. Moving to $P e \sim O(1)$ advection comes into play, and the symmetry is broken. For larger $P e$ the probe acts as a "bulldozer:" it accumulates bath particles in a $O\left(P^{-1}\right)$ thin boundary layer on its upstream side and leaves a wake of bath-particle free suspending fluid behind it. Note, the "width" 

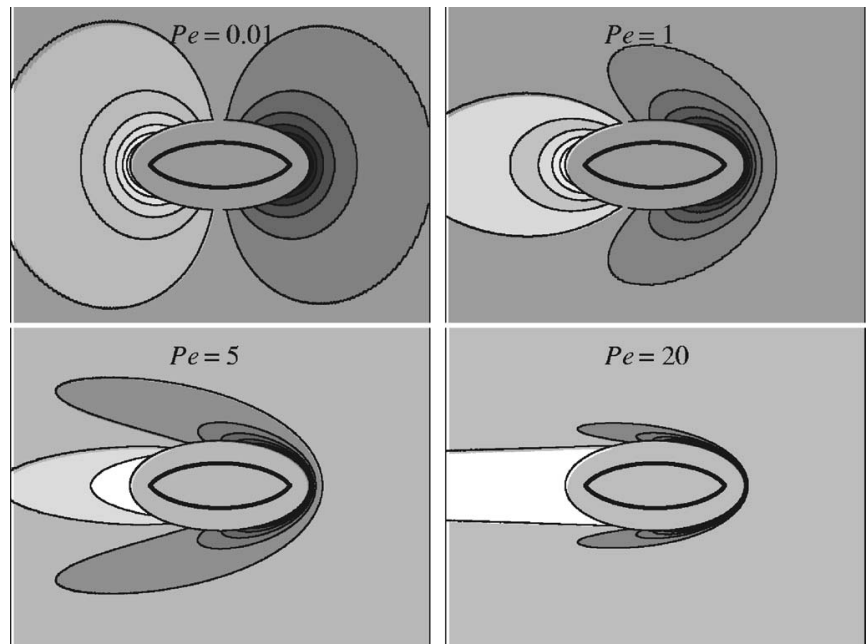

FIG. 10. Microstructural deformation, $g-1$, in the symmetry plane of the prolate probe as a function of $P e$. Here, $\hat{a}=3.5$ and the probe moves from left to right. The excluded-volume surface is shown with zero deformation, $g=1$; darker regions imply accumulation, $g>1$; and lighter regions represent depletion, $g<1$. The closed curve inside the excluded-volume surface is the probe itself.

of the boundary layer (i.e., its extent perpendicular to the probe motion) is on the order of the probe's minor semiaxes (which is equal to the bath particle radius $b$, in dimensional terms). Physically speaking, as the probe moves through the dispersion it pushes a single bath particle past it (per unit time).

In Fig. 11 we plot the microstructure around an oblate probe (again, with $\hat{a}=3.5$ ) as a function of $P e$. As per the prolate probe, the deformation is fore-aft symmetric at small $P e$ and exhibits a boundary layer and wake structure at large $P e$. However, the width of the boundary layer at large $P e$ is now on the scale of the probe major semiaxes, $\hat{a}$. Physically, the oblate probe must push a greater (compared to the prolate probe) number
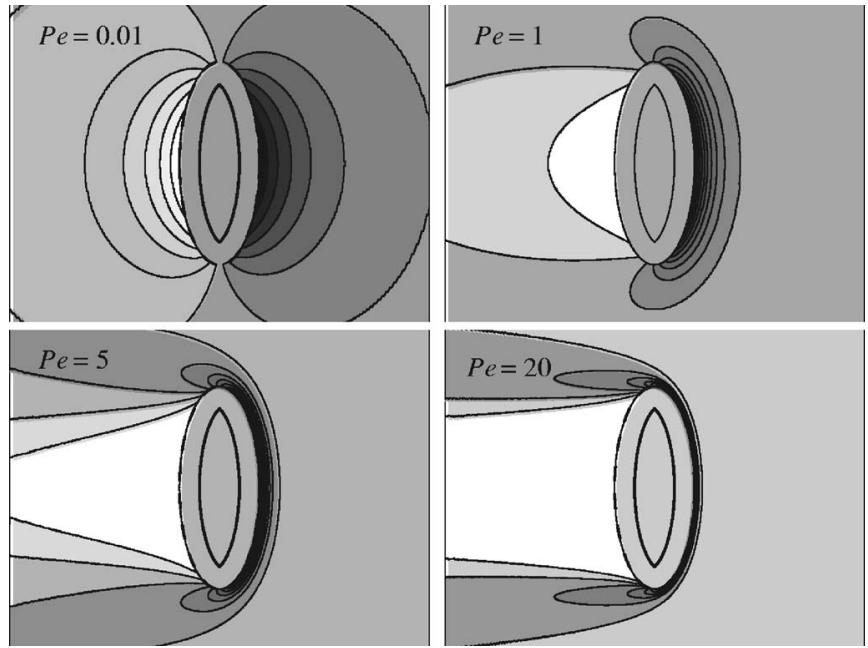

FIG. 11. Microstructural deformation, $g-1$, in the symmetry plane of the oblate probe as a function of $P e$. Here, $\hat{a}=3.5$ and the probe moves from left to right. The shading scheme is the same as in Fig. 10 . 


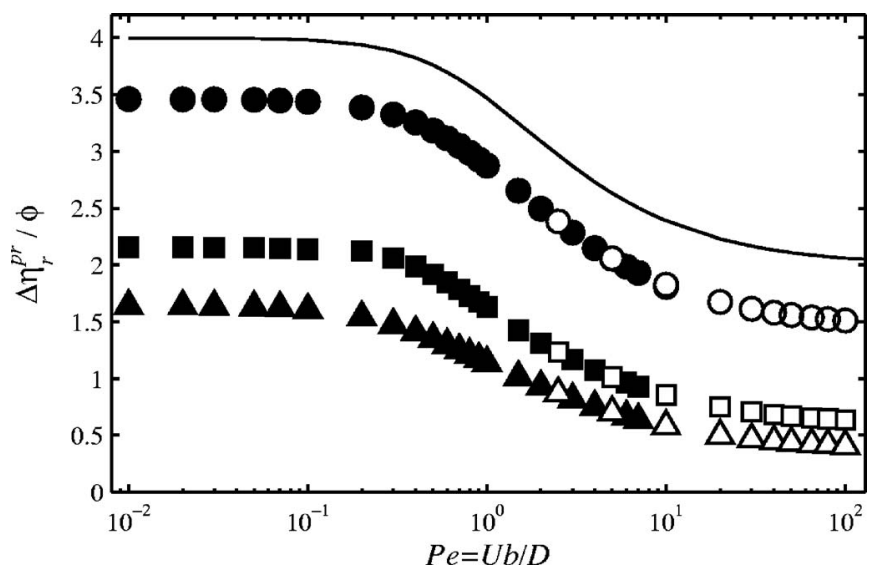

FIG. 12. Microviscosity increment for a prolate probe, $\Delta \eta_{r}^{p r}$, as a function of $P e=U b / D$ for different $\hat{a}=a / b$ : circles, $\hat{a}=1.5$; squares, $\hat{a}=3.5$; and triangles, $\hat{a}=5$. The filling pattern indicates the method of numerical solution: filled, Legendre expansion; and empty, finite differences. The solid line is the microviscosity increment for a spherical probe, $\hat{a}=1$ [Squires and Brady (2005)].

of bath particles out of its path. Consequently, as discussed in Sec. IV B, there is a greater density of bath particles in the boundary layer for the oblate probe, $g^{o b} \sim(\hat{a}+1)^{2} P e$, than the prolate probe, $g^{p r} \sim P e /(\hat{a}+1)$. This disparity in bath-particle density affects fundamentally the microviscosity. Recall, in Sec. III we interpreted the nonequilibrium microstructure around the probe in terms of an osmotic pressure imbalance; the microviscosity increment is the average of the imbalance over the excluded-volume surface. Now, the oblate probe has a bath-particle density of $O\left((\hat{a}+1)^{2} P e\right)$ in its boundary layer and a density of $O(1)$ in the wake behind it. The drop in density occurs over the "edge" of the probe, which is $O(1)(O(b)$ in dimensional terms). Thus, the osmotic pressure gradient that the oblate probe moves against is $O\left((\hat{a}+1)^{2} P e\right)$. In contrast, the prolate probe has a bath-particle density of $O(P e /(\hat{a}+1))$ in its boundary layer and $O(1)$ in its wake, and the drop occurs over the $O(\hat{a}+1)$ length of the probe. Therefore, the osmotic pressure gradient for the prolate probe is $O\left(P e /(\hat{a}+1)^{2}\right)$, which is far smaller than for the oblate probe. Consequently, the average additional external force imposed on the probe (to maintain its steady motion in the face of the osmotic pressure gradient) is far greater in the oblate case. Thus, from the Stokes law definition of the microviscosity, the microviscosity increment inferred by the oblate probe is greater than that by the prolate probe. Next, we examine in detail the microviscosity as a function of $P e$ and $\hat{a}$ for both prolate and oblate probes.

In Fig. 12 we plot the prolate microviscosity increment, $\Delta \eta_{r}^{p r}$, as a function of $P e$ for $\hat{a}=1.5,3.5$, and 5. For each value of $\hat{a}, \Delta \eta_{r}^{p r}$ velocity thins from a Newtonian plateau at small $P e$ to a second Newtonian plateau at large $P e$. This behavior is in qualitative agreement with the spherical probe increment calculated by Squires and Brady (2005). At a given value of $P e, \Delta \eta_{r}^{p r}$ decreases with increasing $\hat{a}$, due to two factors: (i) the upstream contact value of the pair-distribution function decreases; and (ii) the length of the probe (i.e., parallel to the direction of motion) grows. Thus, following the arguments in the paragraph above, the osmotic pressure gradient across $S_{e}$ decreases with increasing $\hat{a}$; consequently, the additional external force, and hence the microviscosity, also decrease.

Ultimately, as $\hat{a} \rightarrow \infty-$ a long thin rod probe-the microviscosity vanishes for all $\mathrm{Pe}$. In this limit, the rod appears infinitely long on the scale of a bath particle, while its 


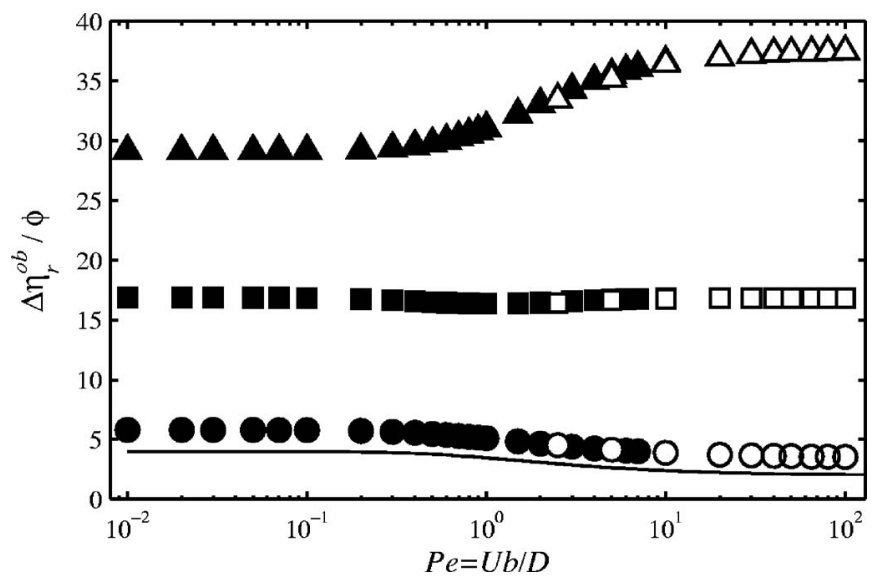

FIG. 13. Microviscosity increment for an oblate probe, $\Delta \eta_{r}^{o b}$, as a function of $P e=U b / D$ for different $\hat{a}$ $=a / b$. The legend is the same as in Fig. 12 .

thickness equals the bath particle radius $b$. Therefore, it must still push a single bath particle out of its path (per unit time), which causes the microstructure around the probe to be deformed. However, as the probe becomes longer ( $\hat{a}$ increases) the osmotic pressure gradient across it becomes weaker and weaker, causing the microviscosity to eventually vanish as $\hat{a} \rightarrow \infty$.

Figure 13 plots the microviscosity increment for the oblate probe, $\Delta \eta_{r}^{o b}$, versus $P e$ for $\hat{a}=1.5,3.5$, and 5. We note for a given value of $P e$ that $\Delta \eta_{r}^{o b}$ increases with increasing $\hat{a}$, which can be explained by the following argument. As $\hat{a}$ increases the area of the excluded-volume surface upon which bath particles from upstream are incident grows $\left(\sim \hat{a}^{2}\right)$. This leads to an increase in the contact value of the pair-distribution function on the upstream side of the probe. Moreover, unlike the prolate probe, as $\hat{a}$ increases the extent of the probe parallel to its motion (i.e., its thickness) stays fixed at 2 ( $2 b$ in dimensional terms). Thus, the osmotic pressure gradient of bath particles across $S_{e}$ increases with increasing $\hat{a}$. As a result, the average external force on the probe, and hence the microviscosity, increase. Finally, as $\hat{a} \rightarrow \infty-$ a thin circular disk probe-the microviscosity diverges for all $P e$ : essentially, the probe must overcome an infinite osmotic pressure gradient to maintain steady motion. In fact, the divergence of the microviscosity is indicative of a breakdown in our theory. Specifically, we assumed a steady microstructure of bath particles is attained; however, as $\hat{a} \rightarrow \infty$, regardless of value of $P e$, it takes an infinite amount of time for a bath particle to pass around from the upstream to downstream side of the probe. Thus, the probe simply builds up an increasing concentration of bath particles on its upstream side and is unable to attain a steady state.

The behavior of the oblate increment for a given value of $\hat{a}$ as a function of $P e$ is far more complicated than the prolate increment. For $\hat{a}=1.5$, in analogy to the spherical and prolate probe microviscosities, the oblate increment monotonically velocity thins from a small $P e$ plateau $\left(\Delta \eta_{r}^{o b} / \phi=5.80\right.$ at $\left.P e=0.001\right)$ to a plateau at high $P e\left(\Delta \eta_{r}^{o b} / \phi=3.55\right.$ at $P e=100)$. However, for $\hat{a}=3.5$ the increment is approximately independent of $P e$ : it undergoes a small amount of velocity thinning initially (from $\Delta \eta_{r}^{o b} / \phi=16.87$ at $P e$ $=0.001$ ), which is recovered by velocity thickening at $P e \sim O(1)$ and greater (at $P e$ $=100$ we find $\left.\Delta \eta_{r}^{o b} / \phi=16.81\right)$. For $\hat{a}=5$ the increment monotonically velocity thickens from a small $P e$ plateau $\left(\Delta \eta_{r}^{o b} / \phi=29.13\right.$ at $\left.P e=0.001\right)$ until a plateau at large $P e$ is reached $\left(\Delta \eta_{r}^{o b} / \phi=37.46\right.$ at $\left.P e=100\right)$. Note, these numerical results are in agreement 
entirely with the asymptotic analysis presented in Sec. IV. (In fact, $\hat{a}=3.5$ was chosen for numerical calculations because it is close to the value, $\hat{a}=3.52$, at which the small and large $P e$ microviscosities are predicted to be equal, cf. Fig. 8.)

At first glance, the transition of $\Delta \eta_{r}^{o b}$ from velocity thinning to thickening with increasing $\hat{a}$ might seem somewhat strange. There is a relatively simple explanation, however. Let us define the microstructural deformation $F^{o b}=g^{o b}-1$. Recall, from Eq. (12) the microviscosity increment is directly proportional to $F^{o b}$ averaged over the excludedvolume surface, $S_{e}$. At small $P e$ the deformation is forced by the no-flux condition at contact $\left[d F^{o b} / d \xi=-(\hat{a}+1) P e \cos \eta\right.$ at $\left.\xi=\xi_{e}\right]$, and the contact value of the deformation was found to be (see Sec. IV A)

$$
F^{o b}(P e \ll 1)=(\hat{a}+1) H\left(\lambda_{e}\right) P e \cos \eta,
$$

where

$$
H\left(\lambda_{e}\right)=\frac{\left(1+\lambda_{e}^{2}\right)^{1 / 2}\left(\lambda_{e} \cot ^{-1} \lambda_{e}-1\right)}{\lambda_{e}\left(1-\lambda_{e} \cot ^{-1} \lambda_{e}\right)-\cot ^{-1} \lambda_{e}} .
$$

The function $H\left(\lambda_{e}\right)=0.5$ for $\hat{a}=1$, and $H\left(\lambda_{e}\right) \rightarrow 0.64$ (to two decimal places) as $\hat{a} \rightarrow \infty$. Hence, to make progress we assume $H\left(\lambda_{e}\right) \approx 0.5$ for all $\hat{a}$. The microstructural deformation averaged over $S_{e}$ is

$$
\left\langle F^{o b}(P e \ll 1)\right\rangle_{S_{e}}=\int_{0}^{\pi} F^{o b}(P e \ll 1) \cos \eta \sin \eta d \eta=2(\hat{a}+1) H\left(\lambda_{e}\right) P e \approx(\hat{a}+1) P e .
$$

At large Pe, from the boundary-layer analysis in Sec. IV B we found

$$
F^{o b}(P e \gg 1)=\frac{1}{4}(\hat{a}+1)^{2} P e \cos \eta ;
$$

importantly, this solution is valid only upstream of the probe, $\eta \leqslant \pi / 2$; downstream there is wake in which $g^{o b} \approx 0$. The surface-averaged deformation is

$$
\left\langle F^{o b}(P e \gg 1)\right\rangle_{S_{e}}=\int_{0}^{\pi / 2} F^{o b}(P e \gg 1) \cos \eta \sin \eta d \eta=\frac{1}{4}(\hat{a}+1)^{2} P e .
$$

The small- and large- $P e$ averaged deformations are equal, $\left(\left\langle F^{o b}(P e \gg 1)\right\rangle_{S_{e}}=\left\langle F^{o b}(P e\right.\right.$ $\ll 1)\rangle_{S_{e}}$ ), at $\hat{a}=3$, which is close to the transition point $\hat{a}=3.52$ of $\Delta \eta_{r}^{o b}$ from velocity thinning to thickening. (Of course, using the full form of $H\left(\lambda_{e}\right)$ would yield the correct value of $\hat{a}=3.52$ from our simple analysis.)

Physically, the transition may be understood from the large $P e$ "flux-balance" argument of Sec. IV B 1. Recall, at steady state the flux of bath particles into the boundary layer on the front of the probe is balanced by a flux out of the boundary layer from the edge of the probe. As $\hat{a}$ increases the probe becomes more oblate, and the influx area grows as $(a+b)^{2}$, while the outflux area grows only linearly with $\hat{a}$, as $(a+b) b$. Thus, increasing $\hat{a}$ leads to a steady state with more and more bath particles in the boundary layer [see Eq. (17)]. Consequently, as $\hat{a}$ increases the probe moves against a stronger osmotic pressure gradient of bath particles, and hence the inferred microviscosity increment increases. Eventually (at $\hat{a}=3.52$ ) the large $P e$ increment exceeds the small $P e$ increment, which gives the transition from velocity thinning to thickening.

Before proceeding to the next section, we pause to make a comment about the microviscosity increment $\Delta \eta_{r}$ as a function of $P e$. As mentioned above, $\Delta \eta_{r}$ is proportional to the contact value of the pair-distribution function, which is $O(P e)$ for all $P e$. And, since there is a factor of $1 / P e$ multiplying the microstructural integrals in Eqs. (11) and (12), 


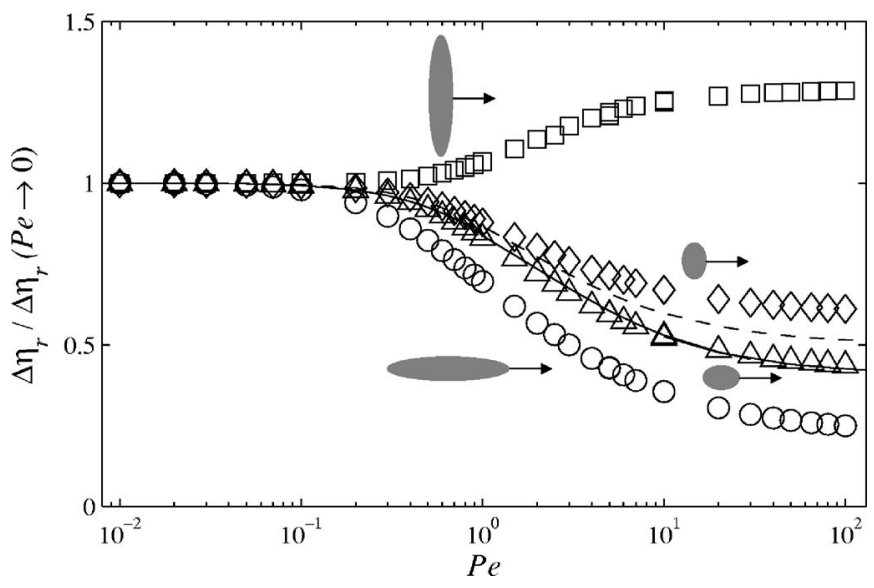

FIG. 14. Comparison of microviscosity increments from prolate and oblate probes with the macroviscosity [Bergenholtz et al. (2002)] and the microviscosity from a spherical probe [Squires and Brady (2005)]. Symbol legend: diamonds, oblate $\hat{a}=1.5$; squares, oblate $\hat{a}=5$; triangles, prolate $\hat{a}=1.5$; circles, prolate $\hat{a}=5$. A sketch of the probe for each case is also shown. The solid line is the macroviscosity and the broken line is the microviscosity for a spherical probe, $\hat{a}=1$. For the microviscosity $P e=U b / D$, and for the macroviscosity $P e$ $=(b \dot{\gamma}) b / D$ (with $\dot{\gamma}$ the shear rate).

$\Delta \eta_{r} \sim O\left(P e^{0}\right)$ for all $P e$. (The microstructural pictures at small and large $P e$ are, of course, very different: for $P e \ll 1$ the probe causes a small $O(P e)$ deformation over a large $O(1 / P e)$ region; in the singular limit of $P e \rightarrow \infty$ there is a large $O(P e)$ deformation in a small $O(1 / P e)$ thin boundary layer.) However, while we know $\Delta \eta_{r}$ is of $O\left(P e^{0}\right)$ at both small and large $P e$, there is no way (without detailed calculation) of knowing in which limit it is greater (and hence if $\Delta \eta_{r}$ thins or thickens with increasing $P e$ ). The thinning/ thickening behavior is dependent on the detailed geometry-in our case the (prolate or oblate) probe shape and $\hat{a}$-of the probe and bath particle configuration. This points up the care that must be taken when selecting a probe (in terms of its shape, size, and orientation) for use in a particular active microrheology experiment.

\section{DISCUSSION}

In this study, we have developed a model for active (nonlinear) microrheology experiments using non-spherical probe particles. We consider the probe to be a body of revolution moving along its symmetry axis at constant velocity, through a colloidal dispersion of spherical bath particles. The probe's shape is such that the excluded volume, or contact, surface between it and a bath particle is a(n) prolate(oblate) spheroid when its major(minor) axis is the axis of revolution. The average external force imposed on the probe (to maintain its steady motion) can be interpreted in terms of a microviscosity of the dispersion via application of Stokes drag law. This is, possibly, the simplest model system one could conceive; however, as shown in the preceding sections, the resulting microrheology is nontrivial.

It is instructive to determine to what extent the results from a particular microrheological experiment represent the true, or macrorheological, properties of a material. As noted by Squires and Brady (2005), for highly complex or unknown materials it is, in general, not possible to compare micro to macro, as the relevant scalings are not known a priori. However, for our model system the scalings are available; thus, we can make a direct, quantitative comparison between the micro- and macro-viscosity. In Fig. 14 we 


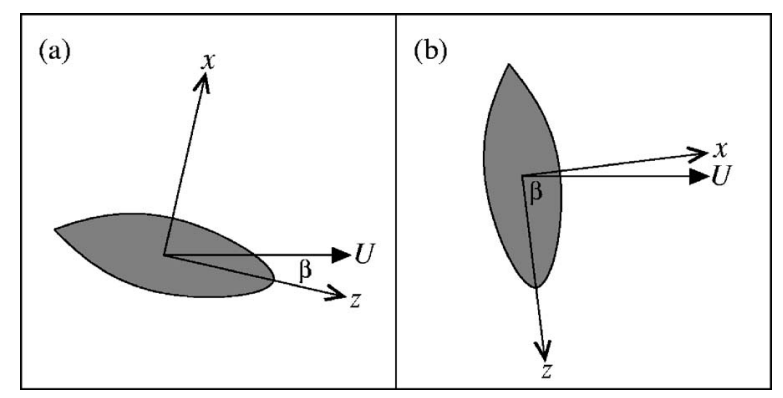

FIG. 15. Sketch of a prolate probe translating at angle $\beta$ to its symmetry axis: (a) near-longwise motion, $\beta$ $\ll 1$; and (b) near-broadside motion, $\pi / 2-\beta \ll 1$. Note, the $y$ axis is directed out of the page.

plot the microviscosity increments for the oblate and prolate probes (for $\hat{a}=1.5$ and $\hat{a}$ =5) versus the macroviscosity [Bergenholtz et al. (2002)] and the microviscosity for a spherical probe [Squires and Brady (2005)]. Note, for macrorheology there is no probe particle, and all particles are spherical with equal radii, $b$. The relative macroviscosity is $\Delta \eta_{r}^{\text {macro }}=1+5 / 2 \phi+\Delta \eta_{r}^{\text {macro }} \phi^{2}$; the macro increment is $O\left(\phi^{2}\right)$, while the micro increment is $O(\phi)$. [The $O(\phi)$ contribution to the macroviscosity is, of course, the single-particle "Einstein correction."] Furthermore, the Péclet number for the macroviscosity is $\mathrm{Pe}$ $=(b \dot{\gamma}) b / D$, where $\dot{\gamma}$ is the shear rate. To aid in the comparison, the macro- and microviscosities have been normalized by their respective limiting values as $P e \rightarrow 0$. For $\hat{a}$ $=1.5$ the oblate and prolate increments velocity thin with increasing $P e$, which qualitatively, and near quantitatively, mimics the "shear thinning" of the macroviscosity. However, for $\hat{a}=5.0$ the oblate increment velocity thickens for all $P e$, which is qualitatively different from the macroviscosity behavior. One should not think, though, this disagreement somehow invalidates the microrheological results. On the contrary, it simply highlights the fundamental physical differences between microrheology and macrorheology [for detailed discussions of these differences, see Squires and Brady (2005) and Khair and Brady (2006)], and emphasizes the care that must be taken when comparing the results from a microrheology experiment using non-spherical probes to the "equivalent" macrorheological measurements.

The theoretical framework presented in this work can be used to study other related problems. A natural extension is to consider a non-spherical (oblate or prolate) probe that is translating with fixed velocity at an angle ( $\beta$, say) to its symmetry axis (see Fig. 15). In this sense, the present study considers the special case $\beta=0$, in which the microstructure is axisymmetric about the direction of motion; however, for non-zero $\beta$ the microstructure is now fully three dimensional. Consequently, to maintain steady motion not only must an additional external force (above the Stokes drag) be imposed on the probe, but also an external torque to prevent the probe from rotating. In Appendix $\mathrm{C}$ we present a preliminary analysis of this problem for small and large $P e$. In the linear-response regime $(P e \ll 1)$ the symmetry of the $O(P e)$ microstructural deformation $(\mathrm{C} 2)$ dictates that the dispersion does not exert a torque on the probe. In contrast, far from equilibrium $(P e \gg 1)$ the highly nonlinear microstructure does necessitate the application of an external torque. Importantly, we find that the external torque is directed perpendicular to the plane of motion; therefore, there is no tendency for the probe to rotate out of that plane or about its symmetry axis. Furthermore, for longwise, $\beta=0$, and broadside, $\beta=\pi / 2$, motion the external torque vanishes, indicating that both are steady modes of translation. However, for near-longwise, $\beta \ll 1$, and near-broadside, $\pi / 2-\beta \ll 1$, motion the disper- 
sion exerts a torque that generates a clockwise rotation. Therefore, the longwise(broadside) translation is an unstable(stable) equilibrium. Moreover, we conjecture that these are the only two equilibrium modes; hence, if $\beta \neq 0$ the rod will adopt a terminal broadside orientation in the absence of an external torque.

Could the external torque on the probe be used to infer more than just a microviscosity? To address this question, we note that Leal (1975) studied the motion of a slender $(\hat{a} \gg 1)$ axisymmetric rod translating in a second-order fluid. The second-order fluid is the second "term" in an asymptotic expansion of the stress of a viscoelastic fluid in the limit of slow and slowly varying flow (the first term is simply the Newtonian stress), which includes first $\left(N_{1}\right)$ and second $\left(N_{2}\right)$ normal stress differences. Like us, Leal (1975) finds that the second-order fluid exerts a torque that is out of the rod's plane of motion; moreover, longwise and broadside translation are the only two steady configurationswhich configuration is stable depends on the normal stress differences. The direction of the torque [see Eq. (47) in Leal (1975)] is given by the sign of the quantity $4 N_{1}+N_{2}$ : if $4 N_{1}+N_{2}$ is positive(negative) the longwise(broadside) mode is stable. Recently, this result has been shown to hold for a prolate spheroid of arbitrary $\hat{a}$ for the special case $N_{1}=-2 N_{2}$ [Galdi (2000)].

Our analysis for a prolate probe gave a stable broadside orientation which, assuming that the second-order fluid model is applicable to the hard-sphere colloidal dispersion, suggests that $4 N_{1}+N_{2}<0$ at large $P e$. Is this consistent with macrorheological observations? To answer this question, we note that the microstructure at large $P e$ was found via a simple 'radial-balance approximation' of the full boundary-layer equations (see Appendix C for details), used originally by Brady and Morris (1997) in calculation of the microstructure of a sheared dispersion at large $P e$. From the radial-balance microstructure Brady and Morris (1997) computed the normal stress differences of the dispersion, finding that $N_{1} \equiv 0$ (by the symmetry properties of the approximate boundary-layer equations) and $N_{2}<0$ at large $P e$. This implies that $4 N_{1}+N_{2}$ is indeed negative, in agreement with our microrheological findings that broadside translation is stable. However, note that Bergenholtz et al. (2002) solved numerically the full macrorheological boundary-layer equations and found $N_{1}$ to be "small" and positive at large $P e$; in fact their results give $4 N_{1}+N_{2}>0$, which suggests that the longwise translation should be stable. Clearly, one needs to solve the full microrheological boundary-layer equations (C3) to determine if the broadside mode is stable as a result of the radial-balance approximation (i.e., would a solution of the full equations give a stable longwise translation?). Furthermore, as shown by Zarraga et al. (2000), for non-colloidal dispersions $\left(P e^{-1} \equiv 0\right)$ both $N_{1}$ and $N_{2}$ are proportional to the shear-rate $\dot{\gamma}$, whereas for the second-order fluid $N_{1}$ and $N_{2}$ scale as $\dot{\gamma}^{2}$. Thus, it is not clear that one can use Leal's [Leal (1975)] results to infer normal stress differences from a prolate probe translating at large $P e .^{2}$ Nevertheless, the discussion above has shown that microrheology with non-spherical probes has the potential to give information on normal stress differences.

In this study we supposed, for simplicity, that the minor semiaxes of the probe was equal in size to the bath particle radius. Thus, while this allowed the probe-bath geometry to be specified by a single quantity, the probe aspect ratio $\hat{a}=a / b$, our results do show a combination of shape and size effects. One could, however, generalize our model by

\footnotetext{
${ }^{2}$ However, colloidal dispersions near equilibrium $(P e \ll 1)$ possess normal stress differences, $N_{1}$ and $N_{2}$, that do scale as $\dot{\gamma}^{2}$ [Brady and Vicic (1995)], in accordance with the second-order fluid model. Thus one can use Leal"s analysis to infer normal stress differences for a prolate probe moving at small $\mathrm{Pe}$. Of course, $\mathrm{Pe}$ must be sufficient in magnitude such that the dispersion is out of the linear-response regime, for which there is no torque on the probe.
} 
setting the bath particle radius equal to $c$ (here, $c$ is not to be confused with the scale factor in spheroidal coordinates, of course). Now, the geometry is set by the ratios $\hat{a}_{1}$ $=a / b$ and $\hat{a}_{2}=a / c$. The additional degree of freedom allows one to study several interesting limiting cases: for example, (i) $\hat{a}_{1} \gg 1$ and $\hat{a}_{2} \sim O(1)$ - a flat disk (oblate)/thin rod (prolate) translating past bath particles of comparable size; (ii) $\hat{a}_{1} \gg 1$ and $\hat{a}_{2} \ll 1$-a flat disk (oblate) / thin rod (prolate) moving through a dispersion of much larger bath particles; and (iii) $\hat{a}_{1} \approx 1$ and $\hat{a}_{2}>1-$ a slightly eccentric probe in a dispersion of smaller bath particles. Case (i) is particularly interesting, as while the oblate probe is a flat disk, it now pushes only one bath particle out of its path (per unit time). Hence, one expects the microviscosity inferred from it may not velocity thicken at large $P e$. As interesting as these problems may be, we must, however, leave a detailed analysis of them to a future study.

Recently, Khair and Brady (2005) examined a spherical probe executing smallamplitude oscillations in a colloidal dispersion. The small-amplitude condition requires that the Péclet number be much less than unity; the system is in the linear-response (passive) regime. There is, however, no such restriction on the (appropriately nondimensionalized) oscillation frequency ( $\alpha$, say). In this limit, the microstructural deformation has a component that is in phase with the probe oscillation and a component that is out of phase. Through application of Stokes drag law one can define a complex microviscosity of the dispersion - the real and imaginary parts of which correspond to liquid-like and solid-like response, respectively. In that sense, the oscillating probe may be used to study the "microviscoelasticity" of the dispersion. As a variation on that (and the present) theme, one may consider a non-spherical probe moving parallel to its axis of revolution with a small-amplitude oscillatory velocity. Again, the microstructural response will contain in-phase and out-of-phase components, from which a complex microviscosity can be inferred. For a spherical probe Khair and Brady (2005) demonstrated that the Cox-Merz rule is obeyed to a reasonable degree (see Fig. 4 in that paper). Recall, the Cox-Merz rule states that the frequency dependence of the modulus of the complex microviscosity should be almost identical to the $P e$ dependence of the steady microviscosity. For the oblate probe, it would be interesting to see if the complex microviscosity "frequency thickens" at large $\alpha$, in analogy to the velocity thickening of the nonlinear (steady) microviscosity at large $P e$ for $\hat{a}>3.52$ (see Fig. 13). If the complex microviscosity does not thicken this would constitute a violation of the Cox-Merz rule.

\section{ACKNOWLEDGMENT}

This research was supported in part by Grant No. CTS -0500070 from the National Science Foundation.

\section{APPENDIX A: EXACT SOLUTION OF THE SMOLUCHOWSKI EQUATION}

Here, we derive an exact solution to the Smoluchowski equation (2) in spheroidal coordinates. In the interests of brevity, we deal with the prolate probe only; however, it is possible to calculate an exact solution for the oblate probe also (either from first principles or by simply using the transformation (8) in the formulas below).

First, we write the pair-distribution function as

$$
g^{p r}=1+f^{p r} e^{-c \kappa \cosh \xi \cos \eta}
$$

where $\kappa=P e / 2$. Substituting this into the Smoluchowski equation (7) we find that $f^{p r}$ satisfies a modified Helmholtz equation: 


$$
\frac{1}{\sinh \xi} \frac{\partial}{\partial \xi}\left(\sinh \xi \frac{\partial f^{p r}}{\partial \xi}\right)+\frac{1}{\sin \eta} \frac{\partial}{\partial \eta}\left(\sin \eta \frac{\partial f^{p r}}{\partial \eta}\right)=c^{2} \kappa^{2}\left(\sinh ^{2} \xi+\sin ^{2} \eta\right) f^{p r},
$$

subject to the boundary conditions

$$
\begin{gathered}
\frac{\partial f^{p r}}{\partial \xi}=-2 \kappa \cos \eta\left[1+2 e^{(\hat{a}+1) \kappa \cos \eta}\right] \quad \text { at } \xi=\xi_{e}, \\
f^{p r} \rightarrow 0 \quad \text { as } \xi \rightarrow \infty
\end{gathered}
$$

The solution to (A1) is found by separation of variables [see, e.g., Flammer (1957)] and can be written as an eigenfunction expansion:

$$
f^{p r}=\sum_{n=0}^{\infty} A_{n} S_{0 n}(i k, \mu) R_{0 n}^{(3)}(i k, \tau),
$$

where $\mu=\cos \eta, \tau=\cosh \xi, k=c \kappa$, and the $A_{n}$ are expansion coefficients. The function $S_{0 n}$ is the (axisymmetric) angle function of the first kind and is defined as a sum of Legendre polynomials:

$$
S_{0 n}(i k, \mu)=\sum_{r=0,1}^{\infty} d_{r}^{0 n}(i k) P_{r}(\mu),
$$

where the summation is over even(odd) values of $r$ when $n$ is even(odd). The summation coefficients $d_{r}^{0 n}$ obey a recurrence relation-see Eq. (3.1.4) of Flammer (1957). The function $R_{0 n}^{(3)}$ is known as the radial function of third kind, which vanishes for large $\tau$ (thus satisfying the far-field boundary condition). Formally, $R_{0 n}^{(3)}$ may be written as a summation of spherical Hankel functions of the first kind (denoted as $h_{r}^{(1)}$ )

$$
R_{0 n}^{(3)}(i k, \tau)=\left(\sum_{r=0,1}^{\infty} d_{r}^{0 n}(i k)\right)^{-1} \sum_{r=0,1}^{\infty} i^{r-n} d_{r}^{0 n}(i k) h_{r}^{(1)}(i k \tau) .
$$

To satisfy the boundary condition at contact we require

$$
\sum_{n=0}^{\infty} A_{n} \frac{\partial R_{0 n}^{(3)}}{\partial \xi} \mid S_{\xi_{e}} S_{0 n}(i k, \mu)=-2 \kappa \mu\left[1+2 e^{(\hat{a}+1) \kappa \mu}\right] .
$$

We note that the angle functions satisfy are orthogonal on the interval $[-1,1]$ :

$$
\int_{-1}^{1} S_{0 n}(i k, \mu) S_{0 p}(i k, \mu) d \mu=\delta_{n p} N_{0 n}(k)
$$

the normalization constant $N_{0 n}(k)$ is given by Eq. (3.1.33) of Flammer (1957). Hence, the constants $A_{n}$ are given by

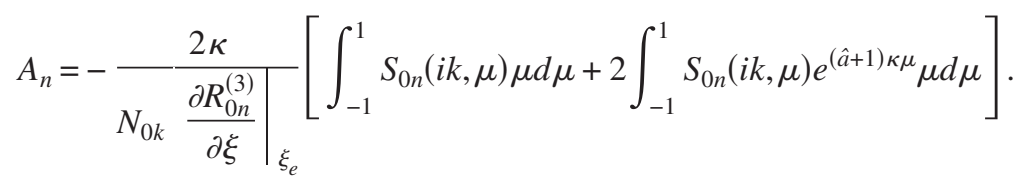

The first integral in Eq. (A2) can be performed analytically; however, the second integral must be computed numerically, which does, unfortunately, limit the usefulness of the exact solution.

Finally, from Eq. (11) the microviscosity increment is given by 


$$
\Delta \eta^{p r}=\frac{6 \phi}{K^{p r} P e} \sum_{n=0}^{\infty} A_{n} R_{0 n}^{(3)}\left(i k, \tau_{e}\right) \int_{-1}^{1} S_{0 n}(i k, \mu) e^{-(\hat{a}+1) \kappa \mu} \mu d \mu .
$$

Again, this integral must be performed numerically.

\section{APPENDIX B: ASYMPTOTIC ANALYSIS AT LARGE Pe}

In this Appendix, we present a rigorous asymptotic analysis of the microstructure at large $P e$, to confirm the findings of our simple physical arguments in Sec. IV B. Detailed calculations are performed for the prolate probe, and the results for the equivalent oblate probe are obtained via the transformation (8).

At large $P e$ advection dominates the microstructure and, except near the probe, the Smoluchowski equation (2) reduces to $\hat{\mathbf{U}} \cdot \boldsymbol{\nabla} g=0$, implying $g$ is constant along a "streamline." The far-field condition (3) dictates this constant to be unity; however, this solution does not satisfy the no-flux boundary condition on the excluded-volume surface $S_{e}(4)$. The Smoluchowski equation is in fact singular in the limit $P e^{-1} \rightarrow 0$, and there exists an "inner" region (or boundary layer) adjacent to the probe in which Brownian diffusion balances advection, thus enabling the no-flux condition on $S_{e}$ to be met.

To focus on the boundary layer we introduce the stretched, or inner, coordinate $y$ $=P e\left(\xi-\xi_{e}\right)$. The Smoluchowski equation in prolate spheroidal coordinates (7) becomes

$$
\begin{aligned}
& \frac{P e^{2}}{\sinh \xi_{e}+P e^{-1} y \cosh \xi_{e}} \frac{\partial}{\partial y}\left[\left(\sinh \xi_{e}+P e^{-1} y \cosh \xi_{e}\right) \frac{\partial g^{p r}}{\partial y}\right]+\frac{1}{\sin \eta} \frac{\partial}{\partial \eta}\left(\sin \eta \frac{\partial g^{p r}}{\partial \eta}\right) \\
& =c P e\left[\left(\cosh \xi_{e}+P e^{-1} y \sinh \xi_{e}\right) \sin \eta \frac{\partial g^{p r}}{\partial \eta}-P e\left(\sinh \xi_{e}+P e^{-1} y \cosh \xi_{e}\right) \cos \eta \frac{\partial g^{p r}}{\partial y}\right],
\end{aligned}
$$

subject to the boundary conditions

$$
\begin{gathered}
\frac{\partial g^{p r}}{\partial y}=-c \sinh \xi_{e} \cos \eta g^{p r} \quad \text { at } y=0 . \\
g^{p r} \rightarrow 1 \quad \text { as } y \rightarrow \infty .
\end{gathered}
$$

Inside the boundary layer we expand the pair-distribution function as $g^{p r}(y, \eta ; P e)$ $=g_{1}^{p r}(y, \eta) P e+g_{2}^{p r}(y, \eta)+O\left(P e^{-1}\right)$. Inserting this expansion into Eq. (B1) yields equations for $g_{1}^{p r}$ and $g_{2}^{p r}$. For $g_{1}^{p r}$ we have

$$
\begin{gathered}
\frac{\partial^{2} g_{1}^{p r}}{\partial y^{2}}+c \sinh \xi_{e} \cos \eta \frac{\partial g_{1}^{p r}}{\partial y}=0, \\
\frac{\partial g_{1}^{p r}}{\partial y}+c \sinh \xi_{e} \cos \eta g_{1}^{p r}=0 \quad \text { at } y=0, \\
g_{1}^{p r} \rightarrow 0 \quad \text { as } y \rightarrow \infty,
\end{gathered}
$$

which has the solution

$$
g_{1}^{p r}(y, \eta)=A(\eta) e^{-c y \sinh \xi_{e} \cos \eta} .
$$

The "angular" function $A(\eta)$ will be found at the next order. Note, this solution is only valid upstream of the probe $(0 \leqslant \eta \leqslant \pi / 2)$, where advection balances diffusion in the 
boundary layer. Downstream of the probe, $\pi / 2 \leqslant \eta \leqslant \pi$, bath particles are advected away and there exists a wake in which $g \approx 0$.

The system for $g_{2}^{p r}$ reads

$$
\begin{gathered}
\frac{\partial^{2} g_{2}^{p r}}{\partial y^{2}}+c \sinh \xi_{e} \cos \eta \frac{\partial g_{2}^{p r}}{\partial y}=c \cosh \xi_{e}\left(\sin \eta \frac{\partial g_{1}^{p r}}{\partial \eta}-y \cos \eta \frac{\partial g_{1}^{p r}}{\partial y}\right)-\operatorname{coth} \xi_{e} \frac{\partial g_{1}^{p r}}{\partial y}, \\
\frac{\partial g_{2}^{p r}}{\partial y}+c \sinh \xi_{e} \cos \eta g_{2}^{p r}=0 \quad \text { at } y=0 . \\
g_{2}^{p r} \rightarrow 1 \quad \text { as } y \rightarrow \infty .
\end{gathered}
$$

The far-field condition on $g_{2}^{p r}$ ensures correct matching to the advective outer solution of unity. Some straightforward working yields

$$
g_{2}^{p r}(y, \eta)=\left[\beta_{1}(\eta)+\beta_{2}(\eta) y+\beta_{3}(\eta) y^{2}\right] e^{-c y \sinh \xi_{e} \cos \eta}-\frac{\beta_{2}}{c \sinh \xi_{e} \cos \eta},
$$

where

$$
\begin{gathered}
\beta_{1}(\eta)=-\frac{\operatorname{coth} \xi_{e}}{c \sinh \xi_{e}}\left[\frac{A(\eta)}{\cos ^{3} \eta}+\frac{\tan \eta}{\cos \eta} \frac{d A(\eta)}{d \eta}\right]-\frac{B(\eta)}{c \sinh \xi_{e} \cos \eta} \\
\beta_{2}(\eta)=-\operatorname{coth} \xi_{e}\left[\tan \eta \frac{d A(\eta)}{d \eta}+\left(1+\sec ^{2} \eta\right) A(\eta)\right] \\
\beta_{3}(\eta)=-\frac{c \cosh \xi_{e}}{2 \cos \eta} A(\eta)
\end{gathered}
$$

One can determine the function $B(\eta)$ by continuing the perturbation expansion to the next order in $P e$; this is not, however, required for our current purposes. To satisfy the far-field condition on $g_{2}^{p r}$ requires $\beta_{2}(\eta)=-c \sinh \xi_{e} \cos \eta$, from which we obtain an equation for $A(\eta)$ :

$$
\tan \eta \frac{d A(\eta)}{d \eta}+\left(1+\sec ^{2} \eta\right) A(\eta)=c \sinh \xi_{e} \tanh \xi_{e} \cos \eta,
$$

which has the solution $A(\eta)=\frac{1}{2} c \sinh \xi_{e} \tanh \xi_{e} \cos \eta$. Hence, the pair-distribution function in the boundary layer may be written as

$$
g^{p r}(y, \eta ; P e)=\frac{1}{2} c \sinh \xi_{e} \tanh \xi_{e} \cos \eta e^{-c P e\left(\xi-\xi_{e}\right) \sinh \xi_{e} \cos \eta}+O(1) .
$$

From Eq. (11) we find that at large $P e$ the microviscosity increment is

$$
\Delta \eta_{r}^{p r}=\frac{6 \phi}{K^{p r} P e} \int_{0}^{\pi / 2} g^{p r} \cos \eta \sin \eta d \eta=\frac{4}{\hat{a}+1} \frac{\phi}{K^{p r}}+O\left(P e^{-1}\right)
$$

where we have used the relations $c \sinh \xi_{e}=2$ and $\tanh \xi_{e}=2 /(\hat{a}+1)$.

For the oblate probe, using the transformation (8) on (B2), we find the pair-distribution function in the boundary layer is

$$
g^{o b}(y, \eta ; P e)=\frac{1}{2} c \cosh \xi_{e} \operatorname{coth} \xi_{e} \cos \eta e^{-c P e\left(\xi-\xi_{e}\right) \cosh \xi_{e} \cos \eta}+O(1),
$$

and from Eq. (12) the microviscosity increment is 


$$
\Delta \eta_{r}^{o b}=\frac{3(1+\hat{a})^{2} \phi}{2 K^{o b} P e} \int_{0}^{\pi / 2} g^{o b} \cos \eta \sin \eta d \eta=\frac{(\hat{a}+1)^{4}}{8} \frac{\phi}{K^{o b}}+O(1),
$$

where we have used $c \cosh \xi_{e}=\hat{a}+1$ and $\operatorname{coth} \xi_{e}=(\hat{a}+1) / 2$. Note, both results above are in complete agreement with the simple geometric balance presented in Sec. IV B.

\section{APPENDIX C: PROLATE PROBE TRANSLATING AT AN ANGLE TO ITS SYMMETRY AXIS}

Here, we present an asymptotic analysis for a prolate probe translating at an angle $\beta$ to its symmetry axis (see Fig. 15). The dimensionless velocity of the probe is

$$
\hat{\mathbf{U}}=\cos \beta \hat{\mathbf{z}}+\sin \beta \hat{\mathbf{x}}
$$

In addition to an external force $\left\langle\mathbf{F}^{\text {ext }}\right\rangle$ (9) one must, in general, apply an external torque $\left\langle\mathbf{T}^{e x t}\right\rangle$ to maintain steady motion. The "osmotic force" exerted by the bath particles on an infinitesimal area $d S_{e}$ of the probe is $-n k \operatorname{Tg}(\mathbf{r}) \mathbf{n} d S_{e}$. Therefore, the average external torque about the center of the probe is

$$
\left\langle\mathbf{T}^{e x t}\right\rangle=n k T \oint \mathbf{r} \wedge \mathbf{n} g(\mathbf{r}) d S_{e},
$$

which for the prolate probe reduces to

$$
\frac{\left\langle\mathbf{T}^{e x t}\right\rangle}{6 \eta b^{2} U}=\frac{6 \phi}{P e \sinh ^{2} \xi_{e}} \int_{0}^{2 \pi} \int_{0}^{\pi} g^{p r}(\mathbf{r})(\cos \theta \hat{\mathbf{y}}-\sin \theta \hat{\mathbf{x}}) \sin ^{2} \eta \cos \eta d \eta d \theta .
$$

Here, $\theta$ is the azimuthal coordinate about the $z$ axis. In the linear-response regime $(P e$ $\ll 1)$ we write $g^{p r}=1+P e f^{p r}$, where $f^{p r}$ solves Laplace's equation and vanishes at large distances. The no-flux condition on the excluded volume surface is

$$
\frac{\partial f^{p r}}{\partial \xi}=-c\left(\cos \beta \sinh \xi_{e} \cos \eta+\sin \beta \cosh \xi_{e} \sin \eta \cos \theta\right) \quad \text { at } \xi=\xi_{e},
$$

representing a weighted combination of axisymmetric (along $z$ axis) and nonaxisymmetric (along $x$ axis) forcing. The solution for $f^{p r}$ is found to be

$$
f^{p r}=-\frac{2}{d Q_{1}^{0}(\tau) /\left.d \xi\right|_{\xi_{e}}} \cos \beta \cos \eta Q_{1}^{0}(\tau)-\frac{\hat{a}+1}{d Q_{1}^{1}(\tau) /\left.d \xi\right|_{\xi_{e}}} \sin \beta \sin \eta Q_{1}^{1}(\tau) \cos \theta
$$

where $\tau=\cosh \xi$ and $Q_{n}^{m}(\tau)$ is an associated Legendre polynomial of the second kind. Using Eq. (C2) with Eq. (C1) we find that the external torque is identically zero-as a consequence of the symmetry of microstructure-in the linear-response regime. On increasing $P e$ this fore-aft symmetry will be broken, resulting in a non-zero torque.

We now consider the opposite extreme, $P e \gg 1$, where advection dominates the microstructure. As in Appendix B, the problem is singular in this limit with a boundary layer adjacent to the probe in which diffusion balances advection. Thus, near $S_{e}$ we stretch $\xi$ as $y=P e\left(\xi-\xi_{e}\right)$. The Smoluchowski equation for $g^{p r}$ in stretched coordinates reads 


$$
\begin{aligned}
\frac{\partial^{2} g^{p r}}{\partial y^{2}}+P e^{-1} \operatorname{coth} \xi_{e} \frac{\partial g^{p r}}{\partial y}= & -c \cos \beta\left[\left(\sinh \xi_{e}+P e^{-1} y \cosh \xi_{e}\right) \cos \eta \frac{\partial g^{p r}}{\partial y}\right. \\
& \left.-P e^{-1} \cosh \xi_{e} \sin \eta \frac{\partial g^{p r}}{\partial \eta}\right]-c \sin \beta\left[\left(\cosh \xi_{e}\right.\right. \\
& \left.\left.+P e^{-1} y \sinh \xi_{e}\right) \sin \eta \frac{\partial g^{p r}}{\partial y}+P e^{-1} \sinh \xi_{e} \cos \eta \frac{\partial g^{p r}}{\partial \eta}\right] \\
& +P e^{-1} c \sin \beta \frac{\sinh ^{2} \xi_{e}+\sin ^{2} \eta}{\sinh _{e} \sin \eta} \sin \theta \frac{\partial g^{p r}}{\partial \theta}+O\left(P e^{-2}\right) ;
\end{aligned}
$$

furthermore, $g^{p r}$ must satisfy the boundary conditions

$$
\begin{gathered}
\frac{\partial g^{p r}}{\partial y}=-c\left(\cos \beta \sinh \xi_{e} \cos \eta+\sin \beta \cosh \xi_{e} \sin \eta \cos \theta\right) g^{p r} \quad \text { at } y=0, \\
g^{p r} \rightarrow 1 \quad \text { as } y \rightarrow \infty
\end{gathered}
$$

One can, in principle, solve for $g^{p r}$ by posing the expansion $g^{p r}=P e g_{1}^{p r}+g_{2}^{p r}+O\left(P e^{-1}\right)$ as per Appendix B; however, the resulting algebra is rather cumbersome. Instead, to find $g^{p r}$ we invoke the "radial-balance approximation" used by Brady and Morris (1997) for the microstructure of a sheared suspension at large $P e$. The physical idea is that the competition between "radial" advection and diffusion generates the large gradients of $g^{p r}$ present in the boundary layer. Thus, we form a "leading-order" equation by retaining the $O\left(P e^{0}\right)$ and the $O\left(P e^{-1}\right)$ radial $(\partial / \partial y)$ terms in Eq. (C3), which gives

$$
\frac{\partial^{2} g^{p r}}{\partial y^{2}}+v_{y}\left(1+\frac{1}{v_{y}} P e^{-1} \operatorname{coth} \xi_{e}+P e^{-1} \frac{\partial \ln v_{y}}{\partial \xi_{e}} y\right) \frac{\partial g^{p r}}{\partial y}=0,
$$

where $v_{y}$ is the radial advective velocity in the boundary layer:

$$
v_{y}(\eta, \theta)=c\left(\cos \beta \sinh \xi_{e} \cos \eta+\sin \beta \cosh \xi_{e} \sin \eta \cos \theta\right) .
$$

The solution to Eq. (C6) satisfying Eqs. (C4) and (C5) is

$$
g^{p r}(y)=\frac{1-v_{y} \int_{0}^{y} e^{-s(z)} d z}{1-v_{y} \int_{0}^{\infty} e^{-s(z)} d z},
$$

where $s(z)$ is given by

$$
s(z)=v_{y}\left[\left(1+\frac{1}{v_{y}} P e^{-1} \operatorname{coth} \xi_{e}\right) z+\frac{1}{2} P e^{-1} \frac{\partial \ln v_{y}}{\partial \xi_{e}} z^{2}\right] .
$$

Note, this solution is valid only if $v_{y}>0$ : the locus of points on the excluded-volume surface satisfying $v_{y}=0$ defines the transition between upstream $\left(v_{y}>0\right)$ and downstream $\left(v_{y}<0\right)$ regions. Physically, bath particles are advected from upstream towards the probe, resulting in a boundary layer, while downstream they are advected away from it, giving a low-density wake in which $g^{p r} \approx 0$. We can rewrite $g^{p r}$ as 


$$
g^{p r}(y)=g^{p r}(0)\left[1-v_{y} \int_{0}^{y} e^{-s(z)} d z\right],
$$

and in the limit $P e \rightarrow \infty$ it is found that

$$
\begin{aligned}
g^{p r}(0) & =P e \tanh \xi_{e} v_{y} \\
& =\frac{2 P e}{(\hat{a}+1)}[2 \cos \beta \cos \eta+(\hat{a}+1) \sin \beta \sin \eta \cos \theta],
\end{aligned}
$$

showing that there is an $O(P e)$ accumulation of bath particles in the upstream boundary layer. Note that for $\beta=0$ we recover, modulo a factor of 2 , the contact value for a probe translating along its symmetry axis, cf. Eq. (23).

Substituting the above into Eq. (C1) we can now evaluate the external torque. However, there is a subtlety: the range of $\eta$ in $(\mathrm{C} 1)$ is formally between 0 to $\pi$ (from the upstream to downstream end of the probe), while the contact value (C8) is valid only in the upstream region, from $\eta=0$ to $\eta=\eta_{l}$, where $v_{y}\left(\eta_{l}, \theta\right)=0$. (The interval $\eta<\eta_{l}<\pi$ corresponds to the downstream region in which $g^{p r} \approx 0$.) From Eq. $(\mathrm{C} 7), \eta_{l}$ satisfies the equation

$$
2 \cos \beta \cos \eta_{l}+(\hat{a}+1) \sin \beta \sin \eta_{l} \cos \theta=0 .
$$

To make progress, we focus on the limits of near-longwise $(\beta \ll 1)$ and near-broadside $(\pi / 2-\beta \ll 1)$ translation. For $\beta \ll 1$, the solution to Eq. (C9) is

$$
\eta_{l}=\frac{\pi}{2}+\frac{\hat{a}+1}{2} \beta \cos \theta
$$

i.e., the $O(\beta)$ tilting causes the boundary layer-wake transition to occur slightly downstream of the probe midpoint $(\eta=\pi / 2)$. The external torque for $\beta \ll 1$ is found to be

$$
\frac{\left\langle\mathbf{T}^{e x t}\right\rangle}{6 \pi \eta b^{2} U}=3 \phi \beta\left[\left(\frac{\hat{a}+1}{2}\right)^{2}-1\right] \hat{\mathbf{y}},
$$

where we have used $\sinh ^{2} \xi_{e}=\left[(\hat{a}+1)^{2} / 4-1\right]^{-1}$. Similarly, for $\pi / 2-\beta \ll 1$ we have

$$
\eta_{l}=\pi-\frac{\hat{a}+1}{2}\left(\frac{\pi}{2}-\beta\right) \cos \theta
$$

thus, in near-broadside translation the boundary layer occupies nearly the entire upstream-facing side of the probe, and the resulting torque is

$$
\frac{\left\langle\mathbf{T}^{e x t}\right\rangle}{6 \pi \eta b^{2} U}=\frac{15}{8} \phi\left(\frac{\pi}{2}-\beta\right)^{4}\left(\frac{\hat{a}+1}{2}\right)^{4}\left[\left(\frac{\hat{a}+1}{2}\right)^{2}-1\right] \hat{\mathbf{y}} .
$$

From Eqs. (C10) and (C11) one can make the following deductions. First, for a spherical probe, $\hat{a}=1$, the torque vanishes as expected. Second, the torque is directed along the positive $y$ axis; hence, there is no tendency for the probe to rotate out of its plane of motion (or about its symmetry axis). Third, for longwise, $\beta=0$, and broadside, $\beta=\pi / 2$, motion the torque vanishes, indicating that both of these are possible equilibrium modes of translation. However, by referring to Fig. 15 for near-longwise and nearbroadside motion the external torque rotates the probe anticlockwise; consequently, the dispersion exerts a torque that generates a clockwise rotation. Hence, in the absence of an external torque the longwise(broadside) translation is an unstable(stable) equilibrium 
configuration. Furthermore, we conjecture that these are the only two equilibrium modes; hence, if $\beta \neq 0$ the rod will adopt a terminal broadside orientation.

Finally, we note that in obtaining $(\mathrm{C} 8)$ the $O\left(P e^{-1}\right)$ angular terms in $(\mathrm{C} 3)$ were neglected, while the $O\left(P e^{-1}\right)$ radial terms were retained, thus forming a radial balance with the leading order $\left[O\left(P e^{0}\right)\right]$ terms (C6). This is generally not a valid procedure, as the angular terms in $(\mathrm{C} 3)$ will affect the $O\left(P e^{0}\right)$ angular structure of the pair-distribution function at contact, $g^{p r}(0)$. However, to leading order $[O(P e)]$ the angular structure of the contact value should not be affected by the neglect of the $O\left(P e^{-1}\right)$ angular terms, although the magnitude of $g^{p r}(0)$ might be. [Indeed, this is evident for $\beta=0$, where the radial-balance solution $(\mathrm{C} 8)$ gives a contact value that is identical, modulo a factor of 2 , to the solution of the full boundary-layer equations, (23)]. Therefore, while a solution of the full boundary-layer equations (C3) may well alter the magnitude of the torque in Eqs. (C10) and (C11), we do not expect the sense of rotation to change.

\section{References}

Amblard, F., A. C. Maggs, B. Yurke, A. N. Pargellis, and S. Leibler, "Subdiffusion and anomalous local viscoelasticity in actin networks," Phys. Rev. Lett. 77(21), 4470-4473 (1996).

Atakhorrami, M., G. H. Koenderink, C. F. Schmidt, and F. C. MacKintosh, "Short-time inertial response of viscoelastic fluids: Observation of vortex propagation,” Phys. Rev. Lett. 95(20), 208302 (2005).

Bergenholtz, J., J. F. Brady, and M. Vicic, "The non-Newtonian rheology of dilute colloidal suspensions," J. Fluid Mech. 456, 239-275 (2002).

Brady, J. F., and J. F. Morris, "Microstructure of strongly sheared suspensions and its impact on rheology and diffusion,” J. Fluid Mech. 348, 103-139 (1997).

Brady, J. F., and M. Vicic, "Normal stresses in colloidal dispersions," J. Rheol. 39(3), 545-566 (1995).

Cheng, Z., P. M. Chaikin, and T. G. Mason, "Light streak tracking of optically trapped thin microdisks," Phys. Rev. Lett. 89(10), 108303 (2002).

Cheng, Z., and T. G. Mason, "Rotational diffusion microrheology," Phys. Rev. Lett. 90(1), 018304 (2003).

Daniels, B. R., B. C. Masi, and D. Wirtz, "Probing single-cell micromechanics in vivo: The microrheology of C. elegans developing embryos,” Biophys. J. 90, 4712-4719 (2006).

Flammer, C., Spheroidal Wave Functions (Stanford University Press, Stanford, 1957).

Galdi, G. P., "Slow steady fall of rigid bodies in a second-order fluid," J. Non-Newtonian Fluid Mech. 90, 81-89 (2000).

Gittes, F., B. Schnurr, P. D. Olmsted, F. C. MacKintosh, and C. F. Schimdt, "Microscopic viscoelasticity: Shear moduli of soft materials determined from thermal fluctuations," Phys. Rev. Lett. 79(17), 3286-3289 (1997).

Habdas, P., D. Schaar, A. C. Levitt, and E. R. Weeks, "Forced motion of a probe particle near the colloidal glass transition," Europhys. Lett. 67(3), 477-483 (2004).

Happel, J., and H. Brenner, Low Reynolds Number Hydrodynamics (Prentice-Hall, Englewood Cliffs, NJ, 1965).

Holzwarth, G., K. Bonin, and D. B. Hill, "Forces required of kinesin during processive transport through cytoplasm,” Biophys. J. 82, 1784-1790 (2002).

Joly, L., C. Ybert, and L. Bocquet, "Probing the nanohydrodynamics at liquid-solid interfaces using thermal motion," Phys. Rev. Lett. 96(4), 046101 (2006).

Khair, A. S., and J. F. Brady, "Microviscoelasticity of colloidal dispersions," J. Rheol. 49(6), 1449-1481 (2005).

Khair, A. S., and J. F. Brady, "Single particle motion in colloidal dispersions: A simple model for active and nonlinear microrheology," J. Fluid Mech. 557, 73-117 (2006).

Leal, L. G., "The slow motion of a slender rod-like particle in a second order-fluid," J. Fluid Mech. 69, 305-337 (1975).

MacKintosh, F. C., and C. F. Schimdt, "Microrheology," Curr. Opin. Colloid Interface Sci. 4(4), 300-307 
(1999).

Mahaffy, R. E., C. K. Shih, F. C. MacKintosh, and J. Käs, "Scanning probe-based frequency dependent microrheology of polymer gels and biological cells," Phys. Rev. Lett. 85(4), 880-883 (2000).

Mason, T. G., and D. A. Weitz, "Optical measurements of frequency-dependent linear viscoelastic moduli of complex fluids,” Phys. Rev. Lett. 74(7), 1250-1253 (1995).

Mason, T. G., K. Ganesan, J. H. van Zanten, D. Wirtz, and S. C. Kuo, "Particle tracking microrheology of complex fluids," Phys. Rev. Lett. 79(17), 3282-3285 (1997).

Meyer, A., A. Marshall, B. G. Bush, and E. M. Furst, "Laser tweezer microrheology of a colloidal suspension," J. Rheol. 50(1), 77-92 (2006).

Morse, P. M., and H. Feshbach, Methods of Theoretical Physics. Part II. (McGraw-Hill, New York, 1953).

Hough, L. A., and H. D. Ou-Yang, "A new probe for mechanical testing of nanostructures in soft materials," J. Nanopart. Res. 1, 495-499 (1999).

Sohn, I. S., and R. Rajagopalan, "Microrheology of model quasi-hard-sphere dispersions," J. Rheol. 48(1), 117-140 (2004).

Squires, T. M., and J. F. Brady, "A simple paradigm for active and nonlinear microrheology," Phys. Fluids 17(7), 073101 (2005).

Tu, R. S., and V. Breedveld, "Microrheological detection of protein unfolding," Phys. Rev. E 72(4), 041914 (2005).

Waigh, T. A., "Microrheology of complex fluids," Rep. Prog. Phys. 68, 685-742 (2005).

Wensink, H. H., and H. Löwen, "Rhythmic cluster generation in strongly driven colloidal dispersions," Phys. Rev. Lett. 97(3), 038303 (2006).

Zarraga, I. E., D. A. Hill, and D. T. Leighton, "The characterization of the total stress of concentrated suspensions of noncolloidal spheres in Newtonian fluids," J. Rheol. 44(2), 185-220 (2000). 